\title{
An effect-theoretic reconstruction of quantum theory
}

\author{
John van de Wetering \\ Radboud University Nijmegen, Houtlaan 4, 6525 XZ Nijmegen, Netherlands
}

\begin{abstract}
An often used model for quantum theory is to associate to every physical system a $\mathrm{C}^{*}$-algebra. From a physical point of view it is unclear why operator algebras would form a good description of nature. In this paper, we find a set of physically meaningful assumptions such that any physical theory satisfying these assumptions must embed into the category of finite-dimensional $\mathrm{C}^{*}$-algebras. These assumptions were originally introduced in the setting of effectus theory, a categorical logical framework generalizing classical and quantum logic. As these assumptions have a physical interpretation, this motivates the usage of operator algebras as a model for quantum theory.

In contrast to other reconstructions of quantum theory, we do not start with the framework of generalized probabilistic theories and instead use effect theories where no convex structure and no tensor product needs to be present. The lack of this structure in effectus theory has led to a different notion of pure maps. A map in an effectus is pure when it is a composition of a compression and a filter. These maps satisfy particular universal properties and respectively correspond to 'forgetting' and 'measuring' the validity of an effect.

We define a pure effect theory (PET) to be an effect theory where the pure maps form a dagger-category and filters and compressions are adjoint. We show that any convex finite-dimensional PET must embed into the category of Euclidean Jordan algebras. Moreover, if the PET also has monoidal structure, then we show that it must embed into either the category of real or complex $\mathrm{C}^{*}$-algebras, which completes our reconstruction.
\end{abstract}

\section{Introduction}

Quantum theory may be taken to be the part of quantum physics that deals with physical systems in an abstract way, without referencing any concrete physical implementation. A qubit for instance, is not any particular physical system, but rather is a mathematical description that applies to a class of existing systems. A common way to model a system in quantum theory is to associate a (finite-dimensional) $C^{*}$-algebra to it, such as representing a qubit by $M_{2}(\mathbb{C})$, the set of complex $2 \times 2$ matrices. It is not clear at all at first glance why we should model physical systems using $\mathrm{C}^{*}$-algebras. Could it not be that some other model would work better? Or can we maybe find a reason why we need to use $\mathrm{C}^{*}$-algebras?

Generalised Probabilistic Theories (GPTs) were introduced as a general framework that should be able to fit any physical theory. They are a widely used framework for studying what properties are specific to quantum theory and which are generic [3, 6, 7, 12, 42]. One particular direction of research in which the usage of GPTs is widespread is in studying physical principles from which the structure of quantum theory, $\mathrm{C}^{*}$-algebras, can be derived; a process known as reconstructing quantum theory $[13,17,27,29,30,38,39,46,52]^{1}$. Such physical principles tell us then that any physical theory that is 'well-behaved' enough to have those principles, must be describable by $\mathrm{C}^{*}$-algebras or related structures. Using GPTs, several 'foil' theories have also been found that can be contrasted with quantum theory [11, 23, 40, 43], which in combination with the physical principles found in reconstructions of quantum theory allow us to see more clearly how quantum theory is special and how it is not.

\footnotetext{
John van de Wetering: john@vdwetering.name

${ }^{1}$ Although some reconstructions use a (slightly) different framework [4, 20, 25, 33, 41, 44].
} 
A different framework for studying quantum theory from a broad point of view is that of effectus theory [14]. This is a categorical framework for studying logic in both classical and quantum systems. While notions from GPTs like states and effects are still applicable in an effectus, it generalises the notion of probability by replacing the familiar convex structure by effect algebras. As a result, a wider array of theories where scalars have a more complicated structure are included in its definition, which in principle could include examples like the space-like quantum theory in Ref. [19] where the scalars are the unit interval of a commutative $\mathrm{C}^{*}$-algebra or the quantum theory based on non-standard analysis of Ref. [24] where the scalars are the hyperreal numbers.

As opposed to GPTs, effectus theory started from the ground up in category theory, and in the most general setting does not have a notion of tensor product. As a result, new concepts were developed that could be used in this general setting. In particular, quotients and comprehensions [15] were found to provide a rich structure that allows one to give a new definition of purity for maps $[50,51]$. This definition has proved to be quite fruitful in studying purity in settings beyond finite-dimensional quantum theory [47-49], as their compositional structure seems to be more well-behaved than other ones in use (see Section 3 for an extended discussion on different notions of purity found in the literature). Additionally, by using the notion of an image of a map, a new definition of sharpness can be given for effects.

As the definition of an effectus is quite involved, we will instead use the weaker notion of an effect theory, to be defined later. The main object of interest in this paper is the following:

Definition. A (monoidal) pure effect theory (PET) is a (monoidal) effect theory where

- All effects have filters and compressions.

- All maps have images.

- The negation of a sharp effect is sharp.

- The pure maps form a (monoidal) dagger-category.

- Filters and compressions of a sharp effect are adjoint.

- Compressions of sharp effects are isometries.

The definitions of all the terms can be found in Section 3. Therein it is also illustrated how these, quite mathematically stated, 'axioms' make intuitive sense to hold for a theory of physics.

The main example of a pure effect theory is the category of Euclidean Jordan algebras (EJAs) with positive sub-unital maps [49]. EJAs were one of the first generalisations of quantum theory to be studied [35]. Examples of EJAs include matrix algebras of real, complex or quaternionic self-adjoint matrices (and in fact, this list almost completely exhausts the list of examples [36]). The example of EJAs is in a sense the most general one, which brings us to the main result of this paper:

Theorem (informal). A (non-monoidal) GPT that is a pure effect theory embeds into the category of Euclidean Jordan algebras with positive sub-unital maps.

It should be noted that finding suitable tensor products for EJAs is still an open problem [5], which is why we do not require a tensor product in the above theorem. In fact, it seems that the compositional structure of systems sets regular quantum theory apart from the more general EJAs:

Theorem (informal). A GPT that is a monoidal pure effect theory embeds into either the category of complex $C^{*}$-algebras, or the category of real $C^{*}$-algebras with completely positive sub-unital maps.

The reason we get both real and complex quantum theory in the end, is because we do not require local tomography [31] for monoidal pure effect theories, which is a common way to distinguish the two.

As a monoidal PET in the standard probabilistic setting corresponds to quantum theory in this manner, we can interpret a PET where the probabilities take values in a different set to be a foil for quantum theory in a universe where the rules of probability work differently. This allows for a different perspective than the foil theories developed in the context of GPTs where the convex probabilistic structure is always present. 
The paper is structured as follows. In Section 2 we introduce our framework of effect theories, which is a stripped-down version of an effectus. We also introduce operational effect theories, which are our version of GPTs. Section 3 contains an extended discussion on the different notions of purity that can be found in the literature, and why they are not suitable for our use case, before introducing the version of purity used in effectus theory and the related notions of filters and compressions. In Section 4 we start proving general properties of PETs before transitioning to operational PETs in Section 5 where their relation to Jordan algebras is established. Finally, in Section 6 we study monoidal operational PETs.

\subsection{Comparison to other reconstructions}

There is a variety of reconstructions of quantum theory using a variety of axioms. Nevertheless, some common themes amongst these axioms can be found.

First off is some kind of preservation of purity demand [4, 10, 27, 30, 41, 44] which states that a composition of pure maps (where the definition of pure varies) should again be a pure map. In our setting this corresponds to the requirement that the pure maps should form a category.

Next up is the existence of certain types of filters or the ability to restrict systems to subsystems based on the support of effects or states [4, 13, 27, 30, 44, 52], which in this reconstruction takes the form of asking filters and compressions to exist.

An almost universally used type of axiom that is not present in this paper, is the requirement of some kind of symmetry or convertability between pure states, i.e. the requirement that a certain amount of reversible transformations must exist $[4,13,17,29,30,33,37,39,41,44]{ }^{2}$

Our axiom stating that compressions of sharp effects are isometries is closely related to those compressions being dagger kernels [32]. In Ref. [44] the existence of dagger kernels is assumed as an axiom. The fact that a compression has a one-sided inverse is also related to the ideal compression axiom of Ref. [13]. See [45, Theorem 4.21] for a more formal version of this relation.

The dagger structure axioms for pure maps that we require have no direct comparison in the literature, but it should be noted that in Refs. [41, 44] they require that all maps have a dagger, and particularly the sharp dagger of Ref. [41] is used in a similar way as in this paper, namely to derive symmetry of transition probabilities (Proposition 5.18). The existence of images has no counterpart in the literature ${ }^{3}$.

It is well-established that the existence of a tensor product satisfying local tomography allows one to distinguish arbitrary Euclidean Jordan algebras from complex $C^{*}$-algebras [33, 41, 44, 46, 52] (although some other papers use local tomography in a more substantial way as part of their derivation $[13,29])$. In this paper we use the assumption of the existence of a well-behaved tensorproduct in order to force our systems to be real or complex $\mathrm{C}^{*}$-algebras, a dichotomy that also been found before [5, 28].

Finally, while this paper uses ideas of a general categorical nature, core methods of the proof only work in the setting of GPTs. This is in contrast to Ref. [44] where the structure of the real numbers is only needed at the very end, and the majority of the work is done in the abstract setting of category theory.

\section{Effect Theories}

We start by describing the framework we adopt. This framework is inspired by generalized probabilistic theories, but is more general because we don't yet assume a convex structure on our transformations.

The idea of the framework is to model in an abstract way the things you could possibly do in a lab. To this end our most basic notion is that of a system. This represents any kind of physical object that you could do experiments on. We will denote systems by capital letters $A, B, C, \ldots$

\footnotetext{
${ }^{2}$ In fact, the only reconstructions except for the authors previous work [46] that the author is aware of that do not say anything explicitly about reversible transformations are Refs. [27, 52] which both require the strong assumption of self-duality of the space from the start.

${ }^{3}$ In Ref. [44] maps called 'images' are used, but they are not related to the images of this paper in any direct manner.
} 
We might be able to influence or change a system $A$ in some manner to get a different system $B$. We will denote such transformations by $f: A \rightarrow B$.

Of course we can do transformations one after another, which we will denote by $g \circ f$, and this should satisfy the obvious associativity condition $h \circ(g \circ f)=(h \circ g) \circ f$. Combined with the fact that we of course have the 'trivial' transformation id : $A \rightarrow A$ which simply means we do nothing, we have found the familiar notion of a category.

Next we will assume that we have some kind of special system $I$ that denotes the 'empty' or 'trivial' system. A transformation $\omega: I \rightarrow A$ is then a procedure that creates a system from nothing, i.e. it is a preparation. We will call such transformations states. They represent the different ways in which a system $A$ can be prepared. We define the state space of $A$ to be $\operatorname{St}(A):=\{\omega: I \rightarrow A\}$.

Dually, the transformations $q: A \rightarrow I$ are the ways in which the system $A$ can be destroyed. We call these transformations effects and they model the different types of measurements you can perform on a system $A$. We define the effect space of $A$ to be $\operatorname{Eff}(A):=\{q: A \rightarrow I\}$.

The composition of a state with an effect results in a transformation $q \circ \omega: I \rightarrow I$. Such a transformation from the unit system to itself is called a scalar.

A generalized probabilistic theory fits in this framework. In that case, the states and effects have a convex structure, and the scalars are real numbers from the unit interval. The scalar $q \circ \omega$ is then the probability that a measurement $q$ returns true on a state $\omega$. Instead of this identification of the scalars with the real numbers and a convex structure, we will require a weaker sort of structure, known as an effect algebra. The effect algebra structure will allow us to consider sums and negations of effects.

Definition 2.1. An effect algebra $(E,+, 1,0, \perp)$ is a set $E$ equipped with a partially defined commutative, associative addition operation + (so if $x+y$ is defined then $y+x$ is also defined and $x+y=y+x$. Similar rules hold for associativity), such that

- For all $x, x+0=x$,

- When $x+1$ is defined, then $x=0$,

- For all $x$ there exists a unique $y$ such that $x+y=1$. We denote this $y$ by $x^{\perp}$ or $1-x$ and it is called the complement of $x$.

Remark 2.2. In an effect algebra the addition is automatically cancellative: $x+y=x+z \Longrightarrow$ $y=z$. As a result, it has a natural partial order defined as $x \leq y \Longleftrightarrow \exists z: x+z=y$. This $z$ is unique and will be denoted by $y-x$, so that $x+(y-x)=y$. The complement reverses the order: $x \leq y \Longleftrightarrow y^{\perp} \leq x^{\perp}$.

Effect algebras were originally studied in for instance $[8,21]$ as generalisations of the space of effects in a quantum system $M_{n}(\mathbb{C})$, those being $\operatorname{Eff}\left(M_{n}(\mathbb{C})\right):=\left\{E \in M_{n}(\mathbb{C}) ; 0 \leq E \leq 1\right\}$. This set forms an effect algebra where addition of effects $E$ and $F$ is defined as $E+F$ whenever $E+F \leq 1$. The complement is defined as $E^{\perp}=1-E$. The induced order is the standard one for (positive) matrices. There are many more examples of effect algebras. In particular, the unit interval of any ordered vector space is an effect algebra. Viewing effects as possible measurements, the complement $E^{\perp}$ of an effect $E$ can be seen as negating the measurement outcomes of $E$, and then of course $E+E^{\perp}=1$ gives the measurement that always returns true.

We now have the necessary ingredients to give the formal definition of our framework.

Definition 2.3. An effect theory is a category $C$ with a designated object I that we call the trivial system such that for all objects $A$, its effect space Eff $(A):=\{f: A \rightarrow I\}$ is an effect algebra, and such that every map $g: B \rightarrow A$ preserves addition: $0 \circ g=0$ and $(p+q) \circ g=(p \circ g)+(q \circ g)$ whenever $p+q: A \rightarrow I$ is defined as an effect.

This definition is very general and includes a multitude of structures. In particular, any effectus in partial form [14] is an effect theory. Examples of effectuses include the category of sets with partial maps, the category of von Neumann algebras with normal positive contractive maps and the category of order unit groups. In fact, any biproduct category where a notion of 'contractivity' for a map can be defined results in an effectus [14]. As the effects of an object form an effect 
algebra, we have for each object a special effect 1. In the example of von Neumann algebras, this 1 corresponds to the identity of the algebra. In causal generalized probabilistic theories, it corresponds to the unique causal effect, also known as the (partial) trace.

We will also need to be able to describe composite systems, i.e. given a couple of independent systems $A$ and $B$, it should be possible to describe them as forming one bigger system $A \otimes B$. The structure we wish to use for this is that of a monoidal category.

Definition 2.4. We call an effect theory $(C, I)$ monoidal when $C$ is a monoidal category such that the trivial system $I$ is also the monoidal unit and such that the tensor product preserves the effect algebra structure: $0 \otimes q=0,1 \otimes 1=1$ and for all $q$, whenever $p_{1}+p_{2}$ is defined, $\left(p_{1} \otimes q\right)+\left(p_{2} \otimes q\right)$ is also defined and equal to $\left(p_{1}+p_{2}\right) \otimes q$.

\subsection{Operational Effect Theories}

An important example of an effect theory concerns the category of order unit spaces.

Definition 2.5. An order unit space $(O U S)(V, \leq, 1)$ is a real vector space $V$ partially-ordered $b y \leq$ with a special designated order unit 1 such that for all $a, b, c \in V$ and $\lambda \in \mathbb{R}_{>0}$ :

- $a \leq b \Longleftrightarrow a+c \leq b+c$,

- $a \leq b \Longleftrightarrow \lambda a \leq \lambda b$,

- there exists $n \in \mathbb{N}$ such that $-n 1 \leq a \leq n 1$,

- if $a \leq \frac{1}{n} 1$ for all $n \in \mathbb{N}$ then $a \leq 0$

Definition 2.6. We call a linear map $f: V \rightarrow W$ between order unit spaces positive when $v \geq 0 \Longrightarrow f(v) \geq 0$. It's sub-unital when $f(1) \leq 1$. We denote the category of order unit spaces with positive, sub-unital maps by $\boldsymbol{O U S}$.

The category $\mathbf{O U S}^{\mathrm{op}}$ is an effect theory. The states of this category correspond to positive sub-unital maps $\omega: V \rightarrow \mathbb{R}$, while the effects are positive sub-unital maps $\hat{q}: \mathbb{R} \rightarrow V$. These correspond in the obvious manner to elements $q \in[0,1]_{V}=\{v \in V ; 0 \leq v \leq 1\}$.

The category OUS $^{\text {op }}$ is important, because it is in a sense the most general example of what we call an operational effect theory. Such categories roughly correspond to the widely studied generalized probabilistic theories. Before we give the definition of an operational effect theory, we must introduce a few other concepts.

Definition 2.7. In an effect theory,

- We say the unital states order-separate the effects when for every system $A$ and effects $p, q \in \operatorname{Eff}(A)$ we have $p \leq q$ whenever $p \circ \omega \leq q \circ \omega$ for all $\omega \in \operatorname{St}(A)$ with $1 \circ \omega=1$.

- We say it satisfies local tomography when the effects separate the transformations: for all $f, g: B \rightarrow A$ we have $f=g$ whenever $p \circ f=p \circ g$ for all $p \in \operatorname{Eff}(A)$.

- If it is monoidal we say it satisfies tomography when the effects monoidally separate the transformations: for all $f, g: B \rightarrow A$ we have $f=g$ whenever $p \circ\left(f \otimes i d_{C}\right)=p \circ\left(g \otimes i d_{C}\right)$ for all systems $C$ and $p \in \operatorname{Eff}(A \otimes C)$.

Local tomography tells us that transformations are completely determined by what they do on effects. When monoidal structure is present in the theory, there are more ways to let a transformation interact with an effect, and hence we weaken the definition to (non-local) tomography. The states order-separating the effects essentially tells us that there can be no 'infinitesimal' effects (see Appendix A). These properties allow us to relate effect theories to order unit spaces:

Proposition 2.8. Let $\mathbf{E}$ be an effect theory where the scalars are the real unit interval, and where the unital states separate the effects. Then for all systems $A$ of $\mathbf{E}$, there exists an order unit space $V_{A}$, such that $\operatorname{Eff}(A) \cong[0,1]_{V_{A}}$.

Proof. This proof mostly follows Ref. [34]. For the details we refer to Appendix A. 
The order unit space $V_{A}$ associated to $A$ has its effects associated one-to-one with those of $A$, but this is not necessarily true for the states. Any state $\omega: I \rightarrow A$ can be mapped to a state $\omega^{*}: V_{A} \rightarrow \mathbb{R}$ (see Theorem 2.11 below), but not every state on $V_{A}$ necessarily comes from some state in the effect theory.

Definition 2.9. Let $A$ be a system in an effect theory with real scalars where the unital states separate the effects and let $V_{A}$ be its associated effect space: $[0,1]_{V_{A}} \cong \operatorname{Eff}(A)$.

- We say $A$ is finite-dimensional when $V_{A}$ is.

- We call A state-closed when the collection of unital states $S_{1}(A)$ is closed as a subset of $V_{A}^{*}$ (with respect to the topology induced by the norm).

- We call A scalar-like when $\operatorname{Eff}(A) \cong[0,1]$.

Definition 2.10. A (monoidal) operational effect theory (OET) is a (monoidal) effect theory satisfying the following additional properties.

1. The set of scalars is the real unit interval: $\operatorname{Eff}(I)=[0,1]$.

2. The unital states order-separate the effects.

3. All systems are finite-dimensional.

4. All systems are state-closed.

5. Every scalar-like system is isomorphic to the trivial system.

Note that we do not require an OET to satisfy (local) tomography. As a result, this last condition is needed to prevent the case where we have multiple copies of the trivial system that are taken to be non-isomorphic, and can be seen as a very restricted form of tomography.

Let us compare this definition of an OET to that of a generalized probabilistic theory. In both cases the scalars are of course the real numbers. The order-separation of the effects is similar to operational equivalence in the literature on GPTs [13], but somewhat stronger, as they prevent the presence of infinitesimal effects. The effects forming effect algebras ensures that it is a causal GPT, and furthermore, the effect algebra structure ensures that every effect $q$ has a complement $q^{\perp}$. Though these properties are not implied by the definition of a GPT, they are often assumed as a background assumption [45, Chapter 2]. In the same way, finite-dimensionality and the closure of the state space are standard background assumptions $[4,13]$.

If an OET satisfies local tomography, then all the information in the theory is captured by the structure of the effects:

Theorem 2.11. Let $\mathbb{E}$ be an operational effect theory. Then there is a functor $F: \mathbb{E} \rightarrow \boldsymbol{O U} \boldsymbol{S}^{o p}$ such that $\operatorname{Eff}(A) \cong[0,1]_{F(A)}$. Furthermore, this functor is faithful if and only if $\mathbb{E}$ satisfies local tomography.

Proof. This proof mostly follows Ref. [34]. For the details we refer to Appendix A.

\subsection{Euclidean Jordan algebras}

Euclidean Jordan algebras (EJAs) are a type of algebra originally intended to generalise the space of observables of a quantum system.

Definition 2.12. We call $(V, *, 1)$ a Jordan algebra when $V$ is a real vector space with a bilinear commutative Jordan product $*: V \times V \rightarrow V$ that satisfies the Jordan identity: for all $a, b \in V$ $(a * a) *(b * a)=((a * a) * b) * a$. A Euclidean Jordan algebra (EJA) is a finite-dimensional Jordan algebra equipped furthermore with an inner product satisfying for all $a, b, c \in V\langle a * b, c\rangle=\langle b, a * c\rangle$

Instead of introducing the full theory of EJAs, which is quite involved, we will use the Jordanvon Neumann-Wigner classification theorem to motivate why the EJAs are an interesting class of spaces, and to obviate the need for any in-depth development of the theory. 
Theorem 2.13 (Jordan-von Neumann-Wigner [36]). Any EJA can be written as a direct sum of the following simple EJAs:

- $M_{n}(F)^{\text {sa }}$, the set of $n \times n$ self-adjoint matrices over the field $F$ where $F$ is either the real numbers, the complex numbers or the quaternions. The Jordan product is given by $A * B:=$ $\frac{1}{2}(A B+B A)$ and the inner product is $\langle A, B\rangle:=\operatorname{tr}(A B)$.

- $H \oplus \mathbb{R}$ where $(H,\langle\cdot, \cdot\rangle)$ is a real finite-dimensional Hilbert space. The product is defined by $(v, t) *(w, s)=(s v+t w, t s+\langle v, w\rangle)$ and the inner product is $\langle(v, t),(w, s)\rangle=\langle v, w\rangle+t s$. These spaces are called spin-factors.

- The exceptional Jordan algebra $M_{3}(\mathbb{O})^{\text {sa }}$ of $3 \times 3$ self-adjoint matrices over the octonions, with the Jordan product and inner product analogous to the first case.

Of these possibilities, the spin-factors might seem strangest. Some of them should however be quite familiar. Any ordered vector space for which the state-space is affinely isomorphic to an $n$-dimensional ball is isomorphic to a spin-factor. In particular, the qubit system $M_{2}(\mathbb{C})^{s a}$ is a spin-factor with a 3-dimensional Hilbert space given by the linear span of the Pauli matrices. The spin-factors can therefore be seen as generalisations of the qubit.

From this classification result, it should hopefully be clear why EJAs can be considered 'quantumlike'. Beyond the usual complex $\mathrm{C}^{*}$-algebraic framework, it also allows real and quaternionic systems. EJAs can be organised into a category.

Definition 2.14. Let $V$ be an EJA. We write $a \geq 0$ for $a \in V$ when $\exists b \in V$ such that $a=b * b$. We call a linear map $f: V \rightarrow W$ between EJAs positive when $f(a) \geq 0$ whenever $a \geq 0$. It is sub-unital when $f(1) \leq 1$. We denote the category of EJAs with positive sub-unital maps by $\boldsymbol{E} \boldsymbol{J} \boldsymbol{A}_{p s u}$.

The opposite category $\mathbf{E} \mathbf{J} \mathbf{A}_{\mathrm{psu}}^{\mathrm{op}}$ is an operational effect theory and is remarkably well-behaved (see [49] for more details).

\section{Purity}

As discussed in the introduction, the axioms of our reconstruction mostly concern the specific definition of purity from effectus theory. In order to appreciate this definition, let us first take a broader look at the concept of purity.

In quite a variety of topics in quantum information theory, a notion of purity has proved fruitful $[9,18]$. While there is consensus about which states should be considered pure, when talking about pure maps, the situation is more muddled. There are a variety of different definitions in play that each have their benefits and drawbacks. In this section we will review those different definitions, but first let us consider some properties that would be desirable or expected of an intuitive definition of purity.

First of all, what does it mean to say that a map is 'pure'? In a way, saying that a map is pure is saying that it is 'fundamental' in some way. This can mean multiple things. It could mean that every other map can be made in some way using pure maps, and thus that the pure maps are the basic building blocks of the theory. It could also mean that the pure maps are the only transformations that are part of the fundamental theory, other transformations merely reflecting our ignorance of these 'true' dynamics. For instance, in pure quantum mechanics, the systems are Hilbert spaces, while the only allowed transformations are unitaries. Since this is the fundamental level of the physical theory, all these unitaries can be considered pure. Transitioning to the more general framework of $\mathrm{C}^{*}$-algebras, we also have the liberty to describe classical systems and interactions that do not seem to warrant being called pure, such as the action of throwing away a system by the partial trace map.

We will take the view that a map is pure when it is somehow fundamental to the theory. We will now argue that those maps should form a dagger category.

Definition 3.1. A dagger-category $C$ is a category equipped with an involutive endofunctor $(\cdot)^{\dagger}: C \rightarrow C^{o p}$ that is the identity on objects: $A^{\dagger}=A$. In other words, there is an operation $\dagger$ 
that sends every map $f: A \rightarrow B$ to some map $f^{\dagger}: B \rightarrow A$ such that $\left(f^{\dagger}\right)^{\dagger}=f$, id $d^{\dagger}=i d$ and $(f \circ g)^{\dagger}=g^{\dagger} \circ f^{\dagger}$.

Saying that the pure maps should form a dagger category is in fact stating three different things:

- The identity map is pure. Every physical theory should be able to describe the act of not changing a system.

- The composition of pure maps is pure. If we describe a fundamental set of transformations, then when two transformations could possibly happen after one another, i.e. when they are composable, this combined transformation should also be describable in this fundamental theory and hence be pure.

- The time-reverse of a pure map is pure. We consider the dagger action as describing the reversal of the arrow of time. Saying that the pure maps have a dagger is then akin to saying that for every fundamental operation, the reversed operationa is also fundamental.

If we also wish to describe composite systems, then there is an obvious additional requirement for pure maps that a composite of pure maps should again be pure. In this case the pure maps form a monoidal dagger category ${ }^{4}$.

Now let us go over the definitions of purity found in the literature and see how they compare. Probably the most well-known is that of atomicity and the related notion of convex-extremality used extensively in generalized probabilistic theories [6, 12, 13]. A map $f$ is atomic when any decomposition $f=g_{1}+g_{2}$ implies that $g_{i}=\lambda_{i} f$. The maps $f, g_{1}$ and $g_{2}$ here are then required to be 'sub-causal'. For causal maps one can consider convex-extremality. A map $f$ is convex extreme when any decomposition $f=\lambda g_{1}+(1-\lambda) g_{2}$ for $0<\lambda<1$ implies that $g_{1}=g_{2}=f$. If we take atomicity to be our definition of pure, an immediate problem arises. Consider the $\mathrm{C}^{*}$-algebra $M_{n}(\mathbb{C}) \oplus M_{n}(\mathbb{C})$. The identity can then be written as $\mathrm{id}=\mathrm{id}_{1}+\mathrm{id}_{2}$, and hence it is not atomic and thus not pure. Taking convex-extremality as our definition of purity leads to a more subtle problem: the dagger of convex-extreme maps does not have to be sub-unital.

Other definitions of purity are those given by leaks [41], orthogonal factorizations [16] or dilations [45]. Without going into the details, these definitions of purity are in general not closed under a dagger operation. They also need a tensor product in order to be defined. This makes it impossible to define them when no tensor product is available. On a conceptual level there is then also the issue that the purity of a map using these definitions can only be established by considering external systems and hence purity does not seem to be an inherent property of the system and its dynamics.

It should be noted that all these definitions of purity were specifically designed to be applicable to finite-dimensional systems. When considering for instance von Neumann algebras, it is no longer clear that these definitions serve their intended purpose.

\subsection{Filters and compressions}

Having established that these definitions of purity will not serve for our purposes, we will now introduce the notion of purity arising from effectus theory that we will use for the rest of this paper. This definition basically says that a map is pure when it is a composition of a filter and a compression. A filter corresponds to doing a measurement while a compression corresponds to forgetting such a measurement took place. Taking these maps as fundamental is then rooted in the view of quantum theory as a description of information processing [22].

To understand the definition of a compression, let us consider a system $A$ in an effect theory and let $B$ be a 'subsystem' of $A$, i.e. some system with an embedding map $\pi: B \rightarrow A$. If we have some state $\omega: I \rightarrow B$ on this subsystem, then we can view it as a state on the whole system by 'forgetting' it was actually defined on the subsystem: $\pi \circ \omega: I \rightarrow A$. Now let $q: A \rightarrow I$ be the effect that 'witnesses' whether a state is defined on the subsystem $B$. That is, it is the smallest

\footnotetext{
${ }^{4}$ In a monoidal dagger category it is common to require that the dagger preserves the monoidal structure. We will not need this additional requirement.
} 
effect such that $q \circ \pi \circ \omega=1$ for all states $\omega: I \rightarrow B$. This is the case when $q \circ \pi=1 \circ \pi$. Going in the converse direction, we can for every effect $q$ of $A$ ask which subsystem it witnesses. This will be the subsystem of $A$ where ' $q$ is true'. The map that finds the subsystem of $A$ corresponding to $q$ is what we call a compression for $q$.

Definition 3.2. Let $q: A \rightarrow I$ be an effect in an effect theory. We call a system $\{A \mid q\}$ a compression system for $q$ when there is a map $\pi_{q}:\{A \mid q\} \rightarrow A$ such that $1 \circ \pi_{q}=q \circ \pi_{q}$ that is final with this property: whenever $f: B \rightarrow A$ is such that $1 \circ f=q \circ f$ then there is a unique $\bar{f}: B \rightarrow\{A \mid q\}$ such that the following diagram commutes:

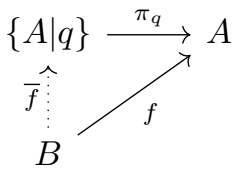

The map $\pi_{q}$ is called a compression for $q^{5}$.

The universal property of compressions tells us that the system $\{A \mid q\}$ is the 'largest' system such that there is a map $\pi:\{A \mid q\} \rightarrow A$ with $1 \circ \pi=q \circ \pi$, and hence this is the system that we should see as the true subsystem that $q$ witnesses.

Example 3.3. Let $A=M_{n}(\mathbb{C})$ and $B=M_{k}(\mathbb{C})$ be complex matrix algebras with $k \leq n$ so that we can view $B$ as a subset of $A$ in the obvious way, i.e. when $\left(c_{i j}\right)$ is matrix in $B$, we can view it as a matrix in $A$ by setting $c_{i j}=0$ when $i, j>k$. Let $\pi: B \rightarrow A$ be this inclusion map, then $\pi$ is a compression for the effect $q \in A$ given by $q=\sum_{i<k}|i\rangle\langle i|$. In fact, it is a compression for any effect $q+p$ where $q$ and $p$ are orthogonal and $\|p\|<1$.

Remark 3.4. It might not be clear from this example why we call such an inclusion map a compression. Originally, compressions were studied in von Neumann algebras [47]. There, unital maps are used. In that case, compressions correspond to certain projection maps. The reason the compression looked like an inclusion in the example is because we used trace-preserving maps instead of unit-preserving maps.

While a compression can be seen as a map that 'forgets' that a state came from a subsystem, a filter is the opposite, describing how a state can be 'filtered' to fit inside a subsystem.

Definition 3.5. Let $q: A \rightarrow I$ be an effect in an effect theory. A filter for $q$ is a map $\xi_{q}: A \rightarrow A_{q}$ such that $1 \circ \xi_{q} \leq q$ which is initial for this property: for any map $f: A \rightarrow B$ which satisfies $1 \circ f \leq q$ there is a unique $\bar{f}: A_{q} \rightarrow B$ such that the following diagram commutes:

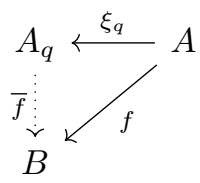

The interpretation of the filter $\xi_{q}$ is that it represents a non-destructive measurement of $q$ which got a positive outcome, i.e. it is a post-selection for $q$. The space $A_{q}$ is the subsystem where $q$ has a nonzero probability of being true.

Example 3.6. Again, let $A=M_{n}(\mathbb{C})$ and $B=M_{k}(\mathbb{C})$ with $k \leq n$ so that we view $B \subseteq A$. Let $q \in A$ be an effect with spectral decomposition $q=\sum_{i} \lambda_{i}\left|v_{i}\right\rangle\left\langle v_{i}\right|$ where the $v_{i}$ are orthogonal and $\left|v_{i}\right\rangle\left\langle v_{i}\right| \in B$ and $\lambda_{i}>0$. We note that then for any matrix $p \in A$ we get $\sqrt{q} p \sqrt{q} \in B$ (when viewed as a subset). Let $\xi_{q}: A \rightarrow B$ be defined by $\xi_{q}(p)=\sqrt{q} p \sqrt{q}$, then $\xi_{q}$ is a filter for $q$.

\footnotetext{
${ }^{5}$ The term 'compression' should not be confused with the maps of the same name in [1]. The compressions from that paper correspond to what we will later call 'assert maps'.
} 
Remark 3.7. In effectus theory, compressions are called comprehensions and filters are called quotients. They arise in a wide variety of settings [15]. For a full account of the theory of effectuses with quotient and comprehension we refer to Ref. [51].

Remark 3.8. Consider the deterministic effect $1: A \rightarrow I$. The subsystem associated with 1 is of course $A$ itself. All the compressions of 1 are then all the ways in which $A$ can be embedded in itself, i.e. they are the isomorphisms of $A$. Isomorphisms of $A$ are also filters for 1 . The filter and compression for the zero effect $0: A \rightarrow I$ is the zero map.

Remark 3.9. Due to their universal properties, the compressions and filters of a given effect are unique up to unique isomorphism. To be more specific, if $\pi:\{A \mid q\} \rightarrow A$ and $f: B \rightarrow A$ are both compressions for $q$, then there is a unique isomorphism $\Theta: B \rightarrow\{A \mid q\}$ such that $f=\pi \circ \Theta$. Similarly, if $\xi_{q}: A \rightarrow A_{q}$ and $g: A \rightarrow B$ are both filters for $q$ then there is a unique isomorphism $\Theta: A_{q} \rightarrow B$ such that $g=\Theta \circ \xi_{q}$. Conversely, if we have an isomorphism, then we can compose it in these ways with a filter or compression to create a new filter or compression.

By these two previous remarks, a filter is more accurately described as a measurement followed by some post-processing in the form of a reversible transformation (and similarly a compression allows some pre-processing).

Using compressions and filters we can define our notion of purity.

Definition 3.10. A map $f: A \rightarrow B$ in an effect theory is pure when $f=\pi \circ \xi$ where $\xi$ is a filter and $\pi$ is a compression.

As a filter corresponds to measuring and post-processing, and a compression corresponds to forgetting, this definition tells us that a map is pure when it can be written in terms of these fundamental operations: a measurement, reversible post-processing, and finally forgetting some information.

Compared to the previous definitions of purity that were discussed, these pure maps are remarkably well-behaved in a variety of settings:

Proposition 3.11. Let $\mathbb{E}$ be $\boldsymbol{v} \boldsymbol{N} \boldsymbol{A}_{n c p s u}$, the category of von Neumann algebras with normal completely positive sub-unital maps or $\boldsymbol{E} \boldsymbol{J} \boldsymbol{A}_{\text {psu }}$, the category of Euclidean Jordan algebras with positive sub-unital maps. Then for every effect in $\mathbb{E}^{o p}$ we can find a compression and a filter and furthermore, the pure maps form a dagger category.

Proof. The von Neumann algebra case can be found in $[47,50]$, while the Jordan algebra case can be found in [49].

\subsection{Sharpness}

As discussed in the previous section, a compression $\pi_{q}:\{A \mid q\} \rightarrow A$ tells us that $\{A \mid q\}$ is a subsystem of $A$. We also know that $1 \circ \pi_{q}=q \circ \pi_{q}$, but $q$ might not be the smallest effect with this property. This is because an effect can be fuzzy, meaning that it does not make a sharp distinction between where it holds true, and where it doesn't. In contrast, if $q$ is the smallest effect with this property, then we call it sharp, since it sharply delineates its subspace.

Definition 3.12. Let $f: A \rightarrow B$ be a transformation in an effect theory. The image of $f$, when it exists, is the smallest effect $q: B \rightarrow I$ such that $q \circ f=1 \circ f$, i.e. if $p: B \rightarrow I$ is also such that $p \circ f=1 \circ f$, then $q \leq p$. We denote the image of $f$ by $i m(f)$.

By the discussion above, we would call an effect sharp when $\operatorname{im}\left(\pi_{q}\right)=q$. Instead we will use the slightly more general formulation used in [51] that turns out to be equivalent (see Proposition 4.6):

Definition 3.13. Let $q: A \rightarrow I$ be an effect. We call $q$ sharp when there is some transformation $f: B \rightarrow A$ such that $\operatorname{im}(f)=q$.

Unfortunately, it seems there is little we can say about sharp effects in a general effect theory, without specifying additional assumptions. Suppose $q$ is a sharp effect. As discussed, this means that there is some subsystem where $q$ is 'true'. Similarly, we would expect there also to be some 
subsystem where $q$ is 'false', i.e. we would expect its negation $q^{\perp}$ to be sharp as well. This is our first assumption.

The second set of assumptions relies on the interplay between filters and compressions of sharp effects. Let $\pi_{q}:\{A \mid q\} \rightarrow A$ be a compression for a sharp effect $q$. Let $\omega: I \rightarrow\{A \mid q\}$ be a state. As the state is part of this subsystem, it satisfies the effect $q$ with certainty. The state $\pi_{q} \circ \omega$ is then the same state on $A$ where we have forgotten that $q$ holds. As $\pi_{q}$ is a pure map, we can consider its adjoint, i.e. the time-reverse, $\pi_{q}^{\dagger}$. As the compression $\pi_{q}$ forgets that the effect $q$ holds for $\omega, \pi_{q}^{\dagger}$ should 'remember it', i.e. it is a post-selection for $q$, and so it is a filter for $q$. But a post-selection after we already knew that the effect holds is just doing nothing, and hence we should have $\pi_{q}^{\dagger} \circ \pi_{q}=\mathrm{id}$. These are our last assumptions. In the parlance of dagger theories: compressions for sharp effects should be isometries, and compressions and filters for sharp effects are adjoint.

\subsection{Pure Effect Theories}

As we now have illustrated all the necessary assumptions, let us define the main object we will study in this paper.

Definition 3.14. A (monoidal) Pure Effect Theory (PET) is a (monoidal) effect theory satisfying the following properties.

(P1) All effects have filters and compressions.

(P2) The pure maps form a (monoidal) dagger-category.

(P3) All maps have images.

(P4) The negation of a sharp effect is sharp (if $q$ is sharp, then $q^{\perp}$ is sharp)

(P5) Filters of sharp effects are adjoint to its compressions (if $\pi_{q}$ is a compression for sharp $q$, then $\pi_{q}^{\dagger}$ is a filter for $q$, and vice versa).

(P6) Compressions of sharp effects are isometries $\left(\pi_{q}^{\dagger} \circ \pi_{q}=i d\right.$ for sharp $\left.q\right)$.

Remark 3.15. All these properties are taken from [51]. They are a subset of what is required of a †-effectus. An effectus satisfying points 1,3 and 4 is called $a \diamond$-effectus. The only known examples of $\dagger$-effectuses are $\boldsymbol{v} \boldsymbol{N} \boldsymbol{A}_{\text {cpsu }}^{\text {op }}[50]$ and $\boldsymbol{E} \boldsymbol{J} \boldsymbol{A}_{\text {psu }}^{\text {op }}[49]$.

Remark 3.16. Several of these properties are closely related to more familiar categorical definitions. It is shown in Ref [51] that an effect theory has all compressions if and only if it has all kernels (a compression of $q$ is a kernel of $q^{\perp}$ ). It has all cokernels if and only if all maps have an image and every sharp effect has a filter (a filter is the cokernel of a compression). The assumptions P5 and P6 can equivalently be stated as "all kernels are dagger-kernels, and the dagger of a kernel is a cokernel", and hence the subcategory of pure maps of a PET is a dagger kernel category [32].

The definition of a PET allows us to formally state our main results:

Theorem (5.24). Let $\mathbb{E}$ be an operational pure effect theory. Then there exists a functor into the opposite category of Euclidean Jordan algebras and sub-unital positive maps $F: \mathbb{E} \rightarrow \boldsymbol{E J}_{\text {psu }}^{\text {op }}$ such that $[0,1]_{F(A)} \cong \operatorname{Eff}(A)$. Furthermore, this functor is faithful if and only if $\mathbb{E}$ satisfies local tomography.

Theorem (6.1). Let $\mathbb{E}$ be a monoidal operational pure effect theory. Then the above functor restricts either to the category of real $C^{*}$-algebras or to the category of complex $C^{*}$-algebras.

In this last theorem we expect that $F$ is faithful if and only if $\mathbb{E}$ satisfies tomography, but showing this requires establishing that $F$ is monoidal, which is currently an open question. For more discussion regarding this we refer to Section 6 . 


\section{Properties of PETs}

In this section we will discuss the basic properties of effect theories, compressions and filters. Let us start with a few results that we will use without further reference:

Proposition 4.1. The following are true in any effect theory.

1. Let $p, q \in \operatorname{Eff}(A)$ be effects such that $p \leq q$, and let $f: B \rightarrow A$ be some map, then $p \circ f \leq q \circ f$.

2. Let $\Theta: A \rightarrow B$ be an isomorphism, i.e. a map that has a 2-sided inverse $\Theta^{-1}: B \rightarrow A$, then $1 \circ \Theta=1$.

Proof.

1. By definition, $p \leq q$ if and only if there is some $r \in \operatorname{Eff}(A)$ such that $p+r=q$. Maps in an effect theory preserve addition and hence $q \circ f=(p+r) \circ f=p \circ f+r \circ f$ so that indeed $p \circ f \leq q \circ f$.

2. Let $1 \circ \Theta=p$. We need to show that $p^{\perp}=0$. Of course $p \circ \Theta^{-1}=1$ and hence $1 \circ \Theta^{-1}=$ $\left(p+p^{\perp}\right) \circ \Theta^{-1}=p \circ \Theta^{-1}+p^{\perp} \circ \Theta^{-1}=1+p^{\perp} \circ \Theta^{-1}$. Since the only effect summable with 1 is 0 , we get $p^{\perp} \circ \Theta^{-1}=0$. As a result $0=0 \circ \Theta=p^{\perp} \circ \Theta^{-1} \circ \Theta=p^{\perp}$ and we are done.

The following proposition contains a few basics results regarding compressions and filters that can be found in Refs. [14, 51].

Proposition 4.2. [14] Let $q: A \rightarrow I$ be an effect and let $\pi_{q}:\{A \mid q\} \rightarrow A$ be a compression for $q$ and $\xi_{q}: A \rightarrow A_{q}$ a filter.

- Let $\Theta: B \rightarrow\{A \mid q\}$ be an isomorphism, then $\pi \circ \Theta$ is another compression for $q$. Conversely, for any two final compressions $\pi$ and $\pi^{\prime}$ for $q$ there is a isomorphism $\Theta$ such that $\pi^{\prime}=\pi \circ \Theta$.

- Let $\Theta: A_{q} \rightarrow B$ be an isomorphism, then $\Theta \circ \xi$ is another filter for $q$. Conversely for any two initial filters $\xi$ and $\xi^{\prime}$ for $q$ there exists a isomorphism $\Theta$ such that $\xi^{\prime}=\Theta \circ \xi$.

- Isomorphisms are filters and compressions for the truth effect 1.

- Zero-maps are filters and compressions for the falsity effect 0.

- As an effect algebra, the set of effects of $A_{q}$ is isomorphic to the downset of $q$ : $\operatorname{Eff}\left(A_{q}\right) \cong$ $\{p \in \operatorname{Eff}(A) ; p \leq q\}$.

Note that all the properties above are relatively straightforward to prove using the universal properties of filters and compressions, except for the last point which takes a bit more work. This last point will be important when we will show the equivalence of atomic and pure effects in Proposition 5.14.

Definition 4.3. We call a transformation $f: A \rightarrow B$ in an effect theory unital when $1 \circ f=1$. We call it faithful when $q \circ f=0$ implies that $q=0$. This is true if and only if $f$ has an image and $\operatorname{im}(f)=1$.

Proposition 4.4. [14, 51] In an effect theory with images, filters and compressions the following are true.

- Compressions are unital.

- Filters are faithful.

- Let $\xi_{q}$ be a filter for $q$, then $1 \circ \xi_{q}=q$.

Definition 4.5. Let $q$ be an effect and let $\pi$ be a compression for $q$. The floor of $q$ is defined as $\lfloor q\rfloor:=i m(\pi)$. The ceiling is defined as the De Morgan dual: $\lceil p\rceil=\left\lfloor p^{\perp}\right\rfloor^{\perp}$.

The ceiling of an effect corresponds to what is called the face of an effect in [13]. In quantum theory, the ceiling of an effect is the projection onto the range of the effect. The floor is the projection onto the space where the effect acts as the identity. 
Proposition 4.6. [51] In an effect theory with images and compressions, the following are true for any effect $q$.

- $\lfloor q\rfloor \leq q$.

- $\lfloor\lfloor\rfloor\rfloor=\lfloor q\rfloor$.

- $p \leq q \Longrightarrow\lfloor p\rfloor \leq\lfloor q\rfloor$.

- $\lceil q\rceil \circ f=0 \Longleftrightarrow q \circ f=0$.

- $\lceil q \circ f\rceil=\lceil\lceil q\rceil \circ f\rceil$.

- $q$ is sharp if and only if $\lfloor q\rfloor=q$.

Note that this proposition implies that the floor of $q$ is the largest sharp predicate below $q$ so that the name is indeed well-chosen.

Proposition 4.7. [51, 208IX] In an effect theory with images, compressions, and filters additionally satisfying $P$, the sharp effects form a lattice.

\subsection{Assert maps}

Let $p$ be a sharp effect and let $\pi_{p}$ be a compression for it. By P5 the map $\pi_{p}^{\dagger}$ is a filter for $p$ and by P6 we have $\pi_{p}^{\dagger} \circ \pi_{p}=$ id.

Definition 4.8. Let $p: A \rightarrow I$ be a sharp effect in a PET and let $\pi_{p}:\{A \mid p\} \rightarrow A$ be a compression for it. We define the assert map asrt $p: A \rightarrow A$ for $p$ by asrt $t_{p}=\pi_{p} \circ \pi_{p}^{\dagger}$.

Remark 4.9. The definition of the assert map does not depend on the choice of compression. If $\pi_{p}^{\prime}$ is also a compression for $p$ then $\pi_{p}^{\prime}=\pi_{p} \circ \Theta_{1}$ for some isomorphism $\Theta_{1}$ by Proposition 4.2, and similarly since $\left(\pi_{p}^{\prime}\right)^{\dagger}$ is a filter by P5 we have $\left(\pi_{p}^{\prime}\right)^{\dagger}=\Theta_{2} \circ \pi_{p}^{\dagger}$ for some other isomorphism $\Theta_{2}$. Now $i d=\left(\pi_{p}^{\prime}\right)^{\dagger} \circ \pi_{p}^{\prime}=\Theta_{2} \circ \pi_{p}^{\dagger} \circ \pi_{p} \circ \Theta_{1}=\Theta_{2} \circ \Theta_{1}$ and hence $\Theta_{2}=\Theta_{1}^{-1}$. As a result $\pi_{p}^{\prime} \circ\left(\pi_{p}^{\prime}\right)^{\dagger}=\pi_{p} \circ \Theta_{1} \circ \Theta_{1}^{-1} \circ \pi_{p}^{\dagger}=\pi_{p} \circ \pi_{p}^{\dagger}=$ asrt $_{p}$.

Remark 4.10. Since $\xi_{p}^{\dagger}$ is also a compression for $p$ we could also define the assert map as asrt ${ }_{p}=$ $\xi_{p}^{\dagger} \circ \xi_{p}$ for any filter of $p$.

Proposition 4.11. [51] Let $p$ be a sharp effect and let $f$ be any composable map in a PET. The following are true:

1. $\operatorname{asrt}_{p} \circ \operatorname{asrt}_{p}=a s r t_{p}$.

2. $\operatorname{im}(\operatorname{asrt} p)=p$.

3. $1 \circ \operatorname{asrt}_{p}=p$.

4. $i m(f) \leq p \Longleftrightarrow$ asrt $_{p} \circ f=f$.

5. $1 \circ f \leq p \Longleftrightarrow f \circ$ asrt $_{p}=f$.

Proof.

1. $\operatorname{asrt}_{p} \circ \operatorname{asrt}_{p}=\pi_{p} \circ \pi_{p}^{\dagger} \circ \pi_{p} \circ \pi_{p}^{\dagger}=\pi_{p} \circ$ id $\circ \pi_{p}^{\dagger}=\operatorname{asrt}_{p}$.

2. Suppose $q \leq \operatorname{im}\left(\operatorname{asrt}_{p}\right)^{\perp}$, then $0=q \circ \operatorname{asrt}_{p}=q \circ \pi_{p} \circ \pi_{p}^{\dagger}$. Because $\pi_{p}^{\dagger}$ is a filter, it is faithful by Proposition 4.4. As a result $q \circ \pi_{p}=0$ so that $q \leq \operatorname{im}\left(\pi_{p}\right)^{\perp}=p^{\perp}$. Taking $q=\operatorname{im}\left(\operatorname{asrt}_{p}\right)^{\perp}$ we then have $p \leq \operatorname{im}\left(\operatorname{asrt}_{p}\right)$. For the other direction we have $p \circ \operatorname{asrt}_{p}=p \circ \pi_{p} \circ \pi_{p}^{\dagger}=1 \circ \pi_{p} \circ \pi_{p}^{\dagger}=$ $1 \circ \operatorname{asrt}_{p}$ so that $p \geq \operatorname{im}\left(\operatorname{asrt}_{p}\right)$.

3. $1 \circ \operatorname{asrt}_{p}=1 \circ \pi_{p} \circ \pi_{p}^{\dagger}=1 \circ \pi_{p}^{\dagger}=p$ by the unitality of $\pi_{p}$ (Proposition 4.4). 
4. If $\operatorname{asrt}_{p} \circ f=f$, then since $1 \circ \operatorname{asrt}_{p} \circ f=p \circ \operatorname{asrt}_{p} \circ f$ we get $\operatorname{im}(f)=\operatorname{im}\left(\operatorname{asrt}_{p} \circ f\right) \leq \operatorname{im}\left(\operatorname{asrt}_{p}\right)=$ $p$. For the other direction, if $\operatorname{im}(f) \leq p$, then $p \circ f=1 \circ f$ so that by the universal property of compressions $f=\pi_{p} \circ \bar{f}$ for some $\bar{f}$. Now $\bar{f}=\operatorname{id} \circ \bar{f}=\pi_{p}^{\dagger} \circ \pi_{p} \circ \bar{f}=\pi_{p}^{\dagger} \circ f$ so that $f=\pi_{p} \circ \bar{f}=\pi_{p} \circ \pi_{p}^{\dagger} \circ f=\operatorname{asrt}_{p} \circ f$.

5. Suppose $f \circ \operatorname{asrt}_{p}=f$, then $1 \circ f=(1 \circ f) \circ \operatorname{asrt}_{p} \leq 1 \circ \operatorname{asrt}_{p}=p$. For the other direction, if $1 \circ f \leq p$, then by the universal property of filters we get $f=\bar{f} \circ \pi_{p}^{\dagger}$ for some $\bar{f}$. Now $\bar{f}=\bar{f} \circ$ id $=\bar{f} \circ \pi_{p}^{\dagger} \circ \pi_{p}=f \circ \pi_{p}$ so that $f=\bar{f} \circ \pi_{p}^{\dagger}=f \circ \pi_{p} \circ \pi_{p}^{\dagger}=f \circ \operatorname{asrt}_{p}$.

Recall that in an effect algebra the addition operation is a partial operation. This will allow us to talk about orthogonality.

Definition 4.12. Let $p, q \in \operatorname{Eff}(A)$ be sharp effects. We call them orthogonal and write $p \perp q$ when $p$ and $q$ are summable. That is, when $p+q$ is defined and therefore $p+q \leq 1$. We call two arbitrary effects (not necessarily sharp) orthogonal when their ceilings are orthogonal: $p \perp q \Longleftrightarrow$ $\lceil p\rceil \perp\lceil q\rceil$.

Note that for non-sharp effects, being summable is weaker than being orthogonal, as for instance $\frac{1}{2} p$ is always summable with itself (assuming we have a scalar acting like $\frac{1}{2}$ ).

Proposition 4.13. Sharp effects $p, q \in E f f(A)$ are orthogonal if and only if $p \leq q^{\perp} \Longleftrightarrow q \leq p^{\perp}$. In a PET $p \perp q$ if and only if $q \circ$ asrt $_{p}=0$.

Proof. Suppose $p+q$ exists. Then there is a $r=(p+q)^{\perp}$ such that $p+q+r=1$, but then by uniqueness of the complement we get $q+r=p^{\perp}$ so that by definition $q \leq p^{\perp}$ (and this argument is obviously symmetric in $p$ and $q$ ). For the other direction, when $q \leq p^{\perp}$ there is an $r$ such that $q+r=p^{\perp}$, but then $q+r+p=p^{\perp}+p=1$. But then $r^{\perp}=q+p$ so that $q$ and $p$ are indeed summable.

Now suppose we have assert maps. We have $\operatorname{im}\left(\operatorname{asrt}_{p}\right)=p$, so that for any $q \leq p^{\perp}=\operatorname{im}\left(\operatorname{asrt}_{p}\right)^{\perp}$ we get $q \circ \operatorname{asrt}_{p} \leq \operatorname{im}\left(\operatorname{asrt}_{p}\right)^{\perp} \circ \operatorname{asrt}_{p}=0$. For the other direction if we have $q \circ \operatorname{asrt}_{p}=0$ then by definition of the image we have $q \leq \operatorname{im}\left(\operatorname{asrt}_{p}\right)^{\perp}=p^{\perp}$.

Proposition 4.14. Let $p, q \in E f f(A)$ be effects in a PET.

1. Let $p, q$ be sharp. If $p \perp q$ then $p+q$ is sharp and is the least upper bound of $p$ and $q$.

2. Suppose $p+q$ is defined and that $p$ and $p+q$ are sharp, then $q$ is sharp.

3. Let $p, q$ be sharp with $p \leq q$, then $q-p$ is sharp.

Proof.

1. Since $p \perp q, p+q$ is again an effect and it is obviously an upper bound of $p$ and $q$. We know that there is a least upper bound ${ }^{6} p \vee q$ by Proposition 4.7. Now since $p \vee q \geq p, q$ we get $p \circ \operatorname{asrt}_{p \vee q}=p$ and $q \circ \operatorname{asrt}_{p \vee q}=q$ by Proposition 4.11 so that $(p+q) \circ \operatorname{asrt}_{p \vee q}=p+q$. Again by Proposition $4.11 p+q=1 \circ(p+q) \leq p \vee q$ so that we must have $p+q=p \vee q$. Since the right-hand side is sharp we are done.

2. $p+q$ is sharp so by $\mathrm{P} 4(p+q)^{\perp}$ is sharp. Since $p \leq p+q$ we have $p^{\perp} \geq(p+q)^{\perp}$ so that $p \perp(p+q)^{\perp}$. By the previous point $p+(p+q)^{\perp}$ is then sharp. Observing that $q+\left(p+(p+q)^{\perp}\right)=1$ we get $q^{\perp}=p+(p+q)^{\perp}$ by the uniqueness of the complement so that $q^{\perp}$ is sharp. Again by P4 we see that $q$ is sharp.

3. By the previous point $p+(q-p)=q$ is sharp so that $q-p$ must be sharp as well.

There is a lot more that can be done in this abstract situation of effect theories with filters and compressions. For the interested reader we refer to Refs. [14, 51]. We will now switch to a more concrete setting.

\footnotetext{
${ }^{6}$ There is a subtlety here where the least upper bound in the set of sharp effects might not be the least upper bound in the set of all effects, but in fact, if $p, q \leq a$ then also $p, q \leq\lfloor a\rfloor$ when $p$ and $q$ are sharp so that the least upper bound in the set of all effects must be sharp, and hence must coincide with the least upper bound in the set of sharp effects.
} 


\section{From operational PETs to Jordan algebras}

In this section we will study PETs in a more familiar convex setting by working with operational PETs. The goal is to show that in this setting, the systems correspond to Euclidean Jordan algebras, and hence most of the structure of quantum theory is recovered. We will first derive a diagonalisation theorem in Section 5.1. Then, in Section 5.2 we will construct an inner product on the effect spaces. In combination with some further technical arguments presented in Appendix B, this will show that the systems correspond to EJAs.

For the duration of this section we will assume that we have fixed some operational PET, and that $A$ and $B$ are systems therein. Furthermore $V$ and $W$ will denote the order unit spaces associated to them: $\operatorname{Eff}(A) \cong[0,1]_{V}$. Sharpness, ceilings and floors in $V$ and $W$ are all defined by the same notion on effects in $A$ and $B$.

\subsection{Diagonalisation}

We will construct a diagonalisation in terms of sharp effects using properties of the ceiling of effects. That is: we will show that for any $v \in V$ we can find a collection of eigenvalues $\lambda_{i} \in \mathbb{R}$ and a set of sharp effects $p_{i} \in V$ that are all orthogonal to each other such that $v=\sum_{i} \lambda_{i} p_{i}$. The following proposition collects the needed properties of the ceiling established in Section 4:

Proposition 5.1. Let $p, q \in E f f(A)$ and $f: B \rightarrow A$.

- If $p \leq q$ then $\lceil p\rceil \leq\lceil q\rceil$ and $\lfloor p\rfloor \leq\lfloor q\rfloor$.

- Let $c>0$ be a scalar. Then $\lceil c p\rceil=\lceil p\rceil$.

- $p \circ f=0$ iff $\lceil p\rceil \circ f=0$.

Proof. All the points are straight from Proposition 4.6 except for $c>0$ implying that $\lceil c p\rceil=\lceil p\rceil$. This follows from the point in Proposition 4.6 that $\lceil q \circ f\rceil=\lceil\lceil q\rceil \circ f\rceil$ by letting $f=p$ and $q=c$ and observing that in our setting $\lceil c\rceil=1$ when the scalar $c$ is greater than zero.

An order unit space has a norm given by $\|v\|:=\inf \{r>0 ;-r 1 \leq v \leq r 1\}$, and this norm induces a topology. Let $C:=\{v \in V ; v \geq 0\}$ be the positive cone of $V$. Its interior with respect to the norm-topology is denoted by $C^{\circ}$. It is straightforward to see that $v \in C^{\circ}$ if and only if there is some $\epsilon>0$ such that $\epsilon 1 \leq v$.

Lemma 5.2. Let $C^{\circ}$ denote the interior of the positive cone of $V$ and $\partial C=C \backslash C^{\circ}$ denote the boundary. Let $v \in C$, then

1. $\|v\| 1-v \in \partial C$.

2. If $\|v\|<1$, then $1-v=v^{\perp} \in C^{\circ}$.

3. If $v$ is sharp, we have $\|v\|<1 \Longrightarrow v=0$.

4. If $\|v\|<1$ and $v \leq p$ where $p$ is sharp, then $\lceil p-v\rceil=p$.

5. If $\|v\|<1$ and $\lceil v\rceil \perp p$ where $p$ is sharp, then $\lfloor p+v\rfloor=p$.

Proof.

1. As $v \leq\|v\| 1$, if $\|v\| 1-v \notin \partial C$ then $v-\|v\| 1 \in C^{\circ}$ so that there must be an $\epsilon>0$ such that $\epsilon 1 \leq v-\|v\| 1$ which means that $v-(\|v\|+\epsilon) 1 \geq 0$ contradicting the defining property of the norm.

2. Of course $0 \leq v \leq\|v\| 1$ and hence $0 \leq 1-\|v\| 1 \leq 1-v=v^{\perp}$. Since $1-\|v\| 1>0$ we conclude that $v^{\perp} \in C^{\circ}$.

3. Let $v$ be sharp with $\|v\|<1$. Then by the previous point $v^{\perp} \in C^{\circ}$ so that $\epsilon 1 \leq v^{\perp}$ for some $\epsilon>0$. Then $1=\lceil 1\rceil=\lceil\epsilon 1\rceil \leq\left\lceil v^{\perp}\right\rceil=v^{\perp}$ because $v^{\perp}$ is sharp. But then $v^{\perp}=1$ so indeed $v=0$. 
4. Let $v \leq p$ with $\|v\|<1$, and denote $q:=\lceil p-v\rceil \leq p$. Then we can write $p=q+r$ where $r=p-q$ is a sharp effect by Proposition 4.14. Now $p-v \leq\lceil p-v\rceil=q$ so that $r=p-q \leq v$. Taking the norm on both sides gives $\|r\| \leq\|v\|<1$ so that by the previous point $r=0$. So indeed $p=q+0=\lceil p-v\rceil$.

5. Note first that because $\lceil v\rceil \perp p$ and hence $\lceil v\rceil+p \leq 1$ we also have $v+p \leq 1$. We calculate $\lfloor p+v\rfloor=\left\lceil(p+v)^{\perp}\right\rceil^{\perp}=\lceil 1-p-v\rceil^{\perp}=\left\lceil p^{\perp}-v\right\rceil^{\perp}$. Since $\lceil v\rceil \perp p$ we have $v \leq\lceil v\rceil \leq p^{\perp}$ and since $\|v\|<1$ the previous point applies and we have $\left\lceil p^{\perp}-v\right\rceil=\left\lceil p^{\perp}\right\rceil$ so that indeed $\lfloor p+v\rfloor=\left\lceil p^{\perp}-v\right\rceil^{\perp}=\left\lceil p^{\perp}\right\rceil^{\perp}=\lfloor p\rfloor=p$.

We need to show that the order unit spaces we deal with are 'finite-rank' in a suitable way. This turns out to follow from the next lemma.

Lemma 5.3. Let $0 \leq v \leq 1$ in $V$. We have $v \in C^{\circ} \Longleftrightarrow\lceil v\rceil=1$.

Proof. First of all, when $v \in C^{\circ}$ we have $\epsilon 1 \leq v$ and hence $\lceil v\rceil \geq\lceil\epsilon 1\rceil=1$, so let us go in the opposite direction.

Let $0 \leq v \leq 1$ and suppose $\lceil v\rceil=1$. We want to show that there exists an $\epsilon>0$ such that $\epsilon 1 \leq v$. Let $\omega$ be a unital state (so that $1 \circ \omega=1$ ) and suppose $v \circ \omega=0$, then $\lceil v\rceil \circ \omega=1 \circ \omega=0$ by the last point of Proposition 5.1. This is of course a contradiction so that $v \circ \omega>0$ for all unital states. Define $f: \operatorname{St}_{1}(A) \rightarrow[0,1]$ by $f(\omega)=v \circ \omega$ where $\operatorname{St}_{1}(A)$ denotes the set of unital states on $A$. By assumption $\operatorname{St}_{1}(A) \subset V_{A}^{*}$ is closed. It is also bounded, and since $V_{A}^{*}$ is a finite-dimensional vector space we conclude that $\operatorname{St}_{1}(A)$ is compact. Therefore the image of $f$ will be some compact subset of $[0,1]$. In particular there is an $\omega$ that achieves the minimum, which has to be strictly bigger than zero: $v \circ \omega \geq \epsilon$. By order-separation we then get $v \geq \epsilon 1$ which shows that $v \in C^{\circ}$.

Remark 5.4. This lemma very explicitly requires the state-space to be closed, and $V$ to be finitedimensional. Many of the following results can be proved without using finite-dimensionality or the closure of state-space if one adds the truth of this lemma to the list of assumptions on our order unit space.

Proposition 5.5. Let $v \in[0,1]_{V}$ with $\|v\|=1$, then $\lfloor v\rfloor \neq 0$.

Proof. By Lemma $5.2\|v\| 1-v \notin C^{\circ}$ and hence by Lemma $5.3\lceil\|v\| 1-v\rceil \neq 1$. Supposing now that $\|v\|=1$ we then immediately get $\lfloor v\rfloor^{\perp}=\left\lceil v^{\perp}\right\rceil=\lceil 1-v\rceil=\lceil\|v\| 1-v\rceil \neq 1$ and hence $\lfloor v\rfloor \neq 0$.

Lemma 5.6. Let $\left\{p_{i}\right\}$ be a finite set of non-zero orthogonal sharp effects (where orthogonal refers to the fact that $p_{i}+p_{j} \leq 1$ if $\left.i \neq j\right)$. Then they are linearly independent.

Proof. Reasoning towards contradiction, assume that there is a non-trivial linear combination of the orthogonal sharp effects. Then without loss of generality $p_{1}=\sum_{i>1} \lambda_{i} p_{i}$. Since all the $p_{i}$ are orthogonal we note that $p_{j} \circ \operatorname{asrt}_{p_{i}}=0$ when $i \neq j$ by Proposition 4.13 , so that $p_{1}=p_{1} \circ \operatorname{asrt}_{p_{1}}=$ $\sum_{i>1} \lambda_{i} p_{i} \operatorname{asrt}_{p_{1}}=0$, a contradiction.

We can now prove our diagonalisation theorem.

Theorem 5.7. Let $v \in[0,1]_{V}$. There is a $k \in \mathbb{N}$ and a strictly decreasing sequence of scalars $\lambda_{1}>\ldots>\lambda_{k}>0$ such that $v=\sum_{i=1}^{k} \lambda_{i} p_{i}$ where the $p_{i}$ are non-zero orthogonal sharp effects.

Proof. If $v=0$ the result is trivial, so assume that $v \neq 0$. Let $v^{\prime}=\|v\|^{-1} v$ so that $\left\|v^{\prime}\right\|=1$. Write $v^{\prime}=\left\lfloor v^{\prime}\right\rfloor+w$ for $w=v^{\prime}-\left\lfloor v^{\prime}\right\rfloor$. By Proposition $5.5\left\lfloor v^{\prime}\right\rfloor \neq 0$. Note that $\|w\| \neq 1$ since otherwise we would get a non-zero $\lfloor w\rfloor$ so that $\lfloor w\rfloor+\left\lfloor v^{\prime}\right\rfloor \leq v^{\prime}$ implying that $\left\lfloor\lfloor w\rfloor+\left\lfloor v^{\prime}\right\rfloor\right\rfloor=\lfloor w\rfloor+\left\lfloor v^{\prime}\right\rfloor \leq\left\lfloor v^{\prime}\right\rfloor$ by Proposition 4.14. Now since $w=v^{\prime}-\left\lfloor v^{\prime}\right\rfloor \leq 1-\left\lfloor v^{\prime}\right\rfloor$ we can write $\lceil w\rceil \leq\left\lceil 1-\left\lfloor v^{\prime}\right\rfloor\right\rceil=\left\lceil\left\lfloor v^{\prime}\right\rfloor^{\perp}\right\rceil=$ $\left\lfloor\left\lfloor v^{\prime}\right\rfloor\right\rfloor^{\perp}=\left\lfloor v^{\prime}\right\rfloor^{\perp}$ so that $\lceil w\rceil \perp\left\lfloor v^{\prime}\right\rfloor$.

Now using $v=\|v\| v^{\prime}$ we see that we can write $v=\lambda_{1} p+w$ where $p$ is a sharp effect, $\lambda_{1}=\|v\|$ and $w$ is an effect orthogonal to $p$ with $\|w\|<\|v\|$. We can now repeat this procedure for $w$. This has to end at some point for if it would not then we get an infinite sequence of orthogonal sharp effects $\left\{p_{i}\right\}_{i=1}^{\infty}$ which by Lemma 5.6 can only be the case when the space is infinite-dimensional.

This diagonalisation is unique in the following sense: 
Proposition 5.8. Let $v=\sum_{i=1}^{k} \lambda_{i} p_{i}$ and $v=\sum_{j=1}^{l} \mu_{j} q_{j}$ where $\lambda_{i}>\lambda_{j}>0$ and $\mu_{i}>\mu_{j}>0$ for $i<j$ and all the $p_{i}$ and $q_{i}$ are sharp and non-zero with all the $p_{i}$ being orthogonal and all the $q_{i}$ being orthogonal. Then $k=l, \lambda_{i}=\mu_{i}$ and $p_{i}=q_{i}$ for all $i$.

Proof. Note first that if $v=0$ that then $k=l=0$ and hence we are done. So assume that $v \neq 0$. It is clear that $\|v\|=\lambda_{1}=\mu_{1}$. Consider $v^{\prime}=\lambda_{1}^{-1} v$. Now $\left\lfloor v^{\prime}\right\rfloor=\left\lfloor p_{1}+\sum_{i>1} \lambda_{1}^{-1} \lambda_{i} p_{i}\right\rfloor=p_{1}$ by application of the last point of Lemma 5.2. Using the second decomposition we also get $\left\lfloor v^{\prime}\right\rfloor=q_{1}$, so that $p_{1}=q_{1}$. Now we can consider $v_{2}=v-\lambda_{1} p_{1}$ and continue the procedure.

We can do this diagonalisation for arbitrary positive elements, because we know that for any positive $a, 0 \leq\|a\|^{-1} a \leq 1$, so that we can diagonalise $\|a\|^{-1} a$ and then rescale. Now for an arbitrary element $a$ (not necessarily positive) we have $-n 1 \leq a \leq n 1$ for some $n$, so that $0 \leq$ $a+n 1 \leq 2 n 1$. This gives us a diagonalisation $a+n 1=\sum_{i} \lambda_{i} p_{i}$, so that $a=\sum_{i} \lambda_{i} p_{i}-n 1=$ $\sum_{i}\left(\lambda_{i}-n\right) p_{i}+n\left(1-\sum_{i} p_{i}\right)$. As a corollary:

Proposition 5.9. Any vector $v \in V$ can be written as $v=v^{+}-v^{-}$where $v^{+}, v^{-} \geq 0$ are orthogonal.

We can get a more finegrained diagonalisation than just the one in terms of sharp effects.

Definition 5.10. We call a non-zero sharp effect $p$ atomic when for all $q$ with $0 \leq q \leq p$ we have $q=\lambda p$. Or in other words when $\downarrow p \cong[0,1]$ where $\downarrow p$ denotes the downset of $p$.

Proposition 5.11. Each non-zero sharp effect can be written as a sum of atomic effects.

Proof. Let $p$ be a non-zero sharp effect. If $p$ is atomic we are done, so suppose it is not. Then we can find an $0 \neq a<p$ such that $a$ is not a multiple of $p$. By the diagonalisation theorem $a=\sum_{i} \lambda_{i} q_{i}$ where the $q_{i}$ are non-zero sharp effects and $0<\lambda_{i} \leq 1$. At least one of these $q_{i}$ is not equal to $p$ since otherwise $a$ would be a multiple of $p$. Pick this $q_{i}$, then obviously $\lambda_{i} q_{i} \leq a \leq p$ and by taking ceilings $q_{i}=\left\lceil q_{i}\right\rceil=\left\lceil\lambda_{i} q_{i}\right\rceil \leq\lceil p\rceil=p$. Since we know that $q_{i} \neq p$ we must have $q_{i}<p$ so that we can write $p=q_{i}+\left(p-q_{i}\right)$, a sum of two orthogonal sharp effects. We can repeat the process for $q_{i}$ and for $p-q_{i}$ which are both smaller sharp effects. By finite-dimensionality and Lemma 5.6 we see that this process has to end at some point, which means at the final step we are only left with atomic effects.

Corollary 5.12. Any $v \in V$ can be written as $v=\sum_{i} \lambda_{i} p_{i}$ where $\lambda_{i} \in \mathbb{R}$ and the $p_{i}$ are atomic and orthogonal.

Proof. First diagonalise $v$ in terms of sharp effects, and then write every sharp effect as a sum of atomic effects.

\subsection{Duality}

In this section we will study pure states and effects and show that they are related trough an interesting duality.

The following proposition establishes that our definition of purity coincides with atomicity when considering states and effects. This correspondence does not hold for arbitrary maps, as there are pure maps that aren't atomic.

Proposition 5.13. An effect $q: A \rightarrow I$ is pure if and only if its corresponding effect $q \in[0,1]_{V}$ is proportional to an atomic effect. A unital state is pure if and only if its image is atomic.

Proof. When $q=0$ this is trivial, so assume that $q \neq 0$.

Suppose $q: A \rightarrow I$ is pure, then it can be written as $q=\pi_{s} \circ \xi_{t}$ where $\xi_{t}: A \rightarrow B$ and $\pi_{s}: B \rightarrow I$. The image $\operatorname{im}(q)$ is a scalar and must be sharp. The only sharp scalars are 0 and 1 , so when $q \neq 0$ we must have $\operatorname{im}(q)=1$ which gives $1=\operatorname{im}\left(\pi_{s} \circ \xi_{t}\right) \leq \operatorname{im}\left(\pi_{s}\right)=s$ so that $s=1$. Compressions for the unit effect are isomorphisms, and hence $\pi_{1}: B \rightarrow I$ is an isomorphism so that $\pi_{1} \circ \xi_{t}=\xi_{t}^{\prime}$ is also a filter and $q=\xi_{t}^{\prime}$. Of course $q=1 \circ q=1 \circ \xi_{t}^{\prime}=t$ so that $t=q$. The filter has type $\xi_{t}^{\prime}: A \rightarrow I$ so that $I \cong A_{q}$ and hence by Proposition $4.2[0,1] \cong \operatorname{Eff}(I) \cong \operatorname{Eff}\left(A_{q}\right) \cong \downarrow q$. As a result $q$ is indeed proportional to an atomic effect. 
For the other direction, use the universal property of filters (see [51, 197VII] for details) to write an effect $q$ as $q=g \circ \xi_{q}$ where $g$ is unital and $\xi_{q}: A \rightarrow A_{q}$. Our goal is to show that $g$ is an isomorphism so that $q$ is indeed pure. First of all, as $g: A_{q} \rightarrow I$ is unital, we have $g=\operatorname{id}_{I} \circ g=1_{I} \circ g=1_{A_{q}}$. Second, since $\downarrow q \cong[0,1]$ by assumption, Proposition 4.2 gives us $\operatorname{Eff}\left(A_{q}\right) \cong \downarrow q \cong[0,1] \cong \operatorname{Eff}(I)$ so that $A_{q}$ is a scalar-like system. Hence by definition of an operational effect theory, there is an isomorphism $\Theta: I \rightarrow A_{q}$. Isomorphisms preserve the unit and hence $g \circ \Theta=1_{A_{q}} \circ \Theta=1_{I}=\mathrm{id}_{I}$, so that $g$ is the inverse of $\Theta$, and hence is an isomorphism. We conclude that $q$ is a composition of a filter and an isomorphism, and hence is pure.

Using a similar sort of argument, when we have a pure state $\omega=\pi_{s} \circ \xi_{t}$ we must have $t=1$ so that $\xi_{1}$ is an isomorphism and then $\operatorname{im}(\omega)=\operatorname{im}\left(\pi_{s}\right)=s$ where $s$ must be proportional to an atomic effect because $[0,1] \cong \operatorname{Eff}(I) \cong \operatorname{Eff}(\{A \mid s\}) \cong \downarrow s$.

Proposition 5.14. Let $q$ be an atomic effect. There exists a unique unital state $\omega_{q}$ such that $\operatorname{im}\left(\omega_{q}\right)=q$. This state is pure and given by $\omega_{q}:=q^{\dagger}$.

Proof. First, for existence: since $q$ is atomic, it is pure by Proposition 5.13, hence $\omega_{q}:=q^{\dagger}$ exists and is a pure state. As also seen in that proof $q=\xi_{q}$, and hence $q^{\dagger}=\xi_{q}^{\dagger}=\pi_{q}$ by P5. Hence $\operatorname{im}\left(\omega_{q}\right)=\operatorname{im}\left(\pi_{q}\right)=q$. Furthermore, since compressions are unital (Proposition 4.4), $\omega_{q}$ is as well.

For uniqueness we note that any unital state $\omega$ can be written as $\pi_{\text {im }(\omega)} \circ \bar{\omega}$ where $\bar{\omega}$ is also a unital state and $\pi_{\operatorname{im}(\omega)}$ is a compression for $\operatorname{im}(\omega)$. Here $\bar{\omega}$ is a map to the object $\{A \mid \operatorname{im}(\omega)\}$ which has $\operatorname{Eff}(\{A \mid \operatorname{im}(\omega)\}) \cong \downarrow \operatorname{im}(\omega)$ by Proposition 4.2. If $\operatorname{im}(\omega)=q$ is atomic then the effect space will be the real numbers so that $\{A \mid \operatorname{im}(\omega)\}$ is a scalar-like system. Hence, using the same argumentation as in the proof of Proposition 5.13, $\bar{\omega}$ will be the unique unital state on this system. This means that any state with $\operatorname{im}(\omega)=q$ will be equal to $\pi_{q}$.

We now have a correspondence between pure states and pure effects. A pure state $\omega$ has an atomic image $q=\operatorname{im}(\omega)$ that is a pure effect. It is the unique pure effect such that $q \circ \omega=1$. In turn $\omega$ is the unique pure state for $q$ such that $q \circ \omega=1$. We want this correspondence to satisfy the following property:

Definition 5.15. We will say an operational PET has symmetry of transition probabilities [1] when for any two atomic effects $p$ and $q$ on the same system we have $q \circ \omega_{p}=p \circ \omega_{q}$ where $\omega_{p}$ is the unique pure unital state with $i m\left(\omega_{p}\right)=p$.

This is easy to show in the following case:

Proposition 5.16. Let $p$ and $q$ be atomic effects. We have $p \circ \omega_{q}=0 \Longleftrightarrow q \circ \omega_{p}=0 \Longleftrightarrow p \perp q$.

Proof. $p \circ \omega_{q}=0 \Longleftrightarrow p \leq \operatorname{im}\left(\omega_{q}\right)^{\perp}=q^{\perp} \Longleftrightarrow p \perp q \Longleftrightarrow q \perp p \Longleftrightarrow q \leq \operatorname{im}\left(\omega_{p}\right)^{\perp} \Longleftrightarrow$ $q \circ \omega_{p}=0$.

The general case is a bit harder to show, and we need the following lemma.

First, note that all scalars are pure maps as they are filters for themselves, and hence the dagger is defined for scalars.

Lemma 5.17. Let $s: I \rightarrow I$ be a scalar, then $s^{\dagger}=s$.

Proof. A scalar $s: I \rightarrow I$ of course corresponds to some number $s \in[0,1]$, and composition of scalars $s \circ t$ is then equal to their product st. We then have $(s t)^{\dagger}=(s \circ t)^{\dagger}=t^{\dagger} \circ s^{\dagger}=s^{\dagger} t^{\dagger}$, so that the dagger preserves multiplication. Note that for real numbers between 0 and 1 we have $s \leq t \Longleftrightarrow \exists r \in[0,1]: s=r t$. As a consequence we get $s \leq t \Longleftrightarrow s=r t \Longleftrightarrow s^{\dagger}=r^{\dagger} t^{\dagger} \Longleftrightarrow$ $s^{\dagger} \leq t^{\dagger}$ so that the dagger is also an order-isomorphism for the unit-interval. Now suppose that $s \leq s^{\dagger}$, then by taking the dagger on both sides we get $s^{\dagger} \leq s$ so that $s=s^{\dagger}$. This of course also holds when we start with $s^{\dagger} \leq s$. Since the unit interval is totally ordered, one of these cases must be true and we are done.

Proposition 5.18. An operational PET has symmetry of transition probabilities. 
Proof. Let $p$ and $q$ be atomic effects. By Proposition 5.13 they are then pure, and to be more specific they are equal to filters: $p=\xi_{p}: A \rightarrow I, q=\xi_{q}: A \rightarrow I$. Since $I$ only has one isomorphism on it, namely the identity, the filters for these effects with this type are unique. Similarly, from Proposition 5.14 we see that their associated pure states $\omega_{p}$ and $\omega_{q}$ are equal to their unique compressions: $\omega_{p}=\pi_{p}$ and $\omega_{q}=\pi_{p}$. Using the dagger we then get $p^{\dagger}=\xi_{p}^{\dagger}=\pi_{p}=\omega_{p}$ and similarly $\omega_{q}^{\dagger}=q$. The expression $q \circ \omega_{p}$ is a scalar and hence by Lemma 5.17 we have $q \circ \omega_{p}=\left(q \circ \omega_{p}\right)^{\dagger}=\omega_{p}^{\dagger} \circ q^{\dagger}=p \circ \omega_{q}$.

Definition 5.19. Let $v, w \in V$ be arbitrary vectors and write them as $v=\sum_{i} \lambda_{i} p_{i}$ and $w=$ $\sum_{j} \mu_{j} q_{j}$, where the $p_{i}$ are orthogonal atoms and the same for the $q_{j}$. Such a decomposition can always be found by Corollary 5.12. Define the inner product of $v$ and $w$ to be $\langle v, w\rangle:=$ $\sum_{i, j} \lambda_{i} \mu_{j}\left(q_{j} \circ \omega_{p_{i}}\right)$.

Proposition 5.20. The inner product defined above is indeed an inner product: well-defined, symmetric and $\langle v, v\rangle \geq 0$ with $\langle v, v\rangle=0$ iff $v=0$.

Proof. First note that with $v$ and $w$ as defined above,

$$
\langle v, w\rangle=\sum_{i, j} \lambda_{i} \mu_{j}\left(q_{j} \circ \omega_{p_{i}}\right)=\sum_{i} \lambda_{i}\left(\sum_{j} \mu_{j} q_{j}\right) \circ \omega_{p_{i}}=\sum_{i} \lambda_{i}\left(w \circ \omega_{p_{i}}\right)
$$

so that the inner product is independent of the representation of $w$ in terms of atomic effects. Second, due to symmetry of transition probabilities $q_{j} \circ \omega_{p_{i}}=p_{i} \circ \omega_{q_{j}}$ and hence $\langle v, w\rangle=\langle w, v\rangle$ so that it is also independent of the representation of $v$.

Finally, we write $\langle v, v\rangle=\sum_{i, j} \lambda_{i} \lambda_{j} p_{j} \circ \omega_{p_{i}}=\sum_{i} \lambda_{i}^{2} p_{i} \omega_{p_{i}}=\sum_{i} \lambda_{i}^{2} \geq 0$ due to Proposition 5.16, and hence $\langle\cdot, \cdot\rangle$ indeed forms an inner product.

Proposition 5.21. The inner-product makes $V$ self-dual: i.e. we have $v \geq 0$ for $v \in V$ if and only if for all $w \geq 0$ we have $\langle v, w\rangle \geq 0$.

Proof. If $v \geq 0$ then we can write $v=\sum_{i} \lambda_{i} p_{i}$ with the $p_{i}$ atomic and orthogonal and $\lambda_{i} \geq 0$ for all $i$. It then easily follows that $\langle v, w\rangle \geq 0$ if $w$ is also positive. For the other direction, suppose $\langle v, w\rangle \geq 0$ for all positive $w$, then in particular $\left\langle v, p_{i}\right\rangle=\lambda_{i} \geq 0$, so that $v$ is indeed positive.

Corollary 5.22. Let $\omega \in S t_{1}(A)$ be a unital state, then $\omega=\sum_{i} \lambda_{i} \omega_{p_{i}}$ with $\lambda_{i} \geq 0, \sum_{i} \lambda_{i}=1$ and the $\omega_{p_{i}}$ being pure states.

Proof. The inner product defines a linear map $f: V \rightarrow V^{*}$ by $f(v)(w):=\langle v, w\rangle$. This map is an injection, so that due to finite-dimensionality it is a bijection. In particular, we can find for every $\omega \in V^{*}$ an element $v \in V$ such that $f(v)=\omega$ and hence $\omega(w)=\langle v, w\rangle$. Since $\omega(w) \geq 0$ for all $w \geq 0$ we must have $v \geq 0$. By expanding $v$ in terms of atomic effects we then get the desired result.

Corollary 5.23. A state is pure if and only if it is convex extremal.

The combination of a self-dual inner product and assert maps brings us very close to the setting of Ref. [1]. In particular by making the appropriate translations we can use their results to prove our main theorem:

Theorem 5.24. Let $\mathbb{E}$ be an operational PET. Then there exists a functor $F: \mathbb{E} \rightarrow \boldsymbol{E J} \boldsymbol{A}_{p s u}^{\text {op }}$ such that for any system $A$ the effect space Eff( $A)$ is isomorphic to the unit interval of its corresponding EJA: $\operatorname{Eff}(A) \cong[0,1]_{F(A)}$. Furthermore, this functor is faithful if and only if $\mathbb{E}$ is locally tomographic.

Proof. By Theorem 2.11 we have a functor $F: \mathbb{E} \rightarrow$ OUS $^{\text {op }}$, so that it suffices to show that the order unit spaces that all systems are mapped to are actually EJAs. We show this by establishing all the conditions of Theorem 9.33 of [1]. Most of the work for this has already been done, but some technical conditions still need to checked. The details can be found in Appendix B. As in Theorem 2.11, faithfulness of the functor corresponds to local tomography in $\mathbb{E}$. 


\section{Monoidal Operational PETs}

We have now shown that systems in an operational PET correspond to Euclidean Jordan algebras. Conversely, the results of Ref. [49] show that the opposite category of EJAs with positive sub-unital maps is an operational PET. Hence, we have characterized operational PETs.

In this section we will see what additional restrictions are imposed on the systems by adding a monoidal structure to the theory that respects the structure of pure maps.

The canonical example of an monoidal operational PET is CStar cpsu $_{\text {op }}$, the opposite category of complex finite-dimensional $\mathrm{C}^{*}$-algebras with completely positive sub-unital maps. Another example is $\mathbf{R S t a r}$ cpsu of real finite-dimensional $\mathrm{C}^{*}$-algebras with completely positive sub-unital maps. A real finite-dimensional $\mathrm{C}^{*}$-algebra is a direct sum of the real matrix algebras $M_{n}(\mathbb{R})$. It turns out that these two examples are the only possibilities.

Theorem 6.1. Let $\mathbb{E}$ be an monoidal operational PET. Then there is a functor $F: \mathbb{E} \rightarrow \mathbb{D}$ where $\mathbb{D}=\boldsymbol{C S t a r}_{c p s u}^{o p}$ or $\mathbb{D}=\boldsymbol{R S t a r}_{c p s u}^{\text {op }}$. This functor preserves the effect space of objects: $\operatorname{Eff}(A) \cong[0,1]_{F(A)}$.

Proof. By Theorem 5.24 we have a functor $F: \mathbb{E} \rightarrow \mathbf{E J A}_{\mathrm{psu}}^{\mathrm{op}}$ so that it suffices to show that the image of this functor lands in the real or complex $\mathrm{C}^{*}$-algebras.

We give a sketch of the proof here, for the details we refer to Appendix C. Note that this proof closely follows the structure of proofs showing how to go from arbitrary EJAs to C*-algebras in Refs. [41, 46].

First we show that composites of atomic effects are again atomic and that the composite preserves orthogonality of atoms. As a result we establish that the composite acts as you would expect with regard to the rank and dimension of the space. We use the argument from Ref. [46] to show that composites of simple EJAs must again be simple. A simple dimension-counting argument establishes that the only simple factors allowed are the real and complex matrix algebras. The final step is to show that it is impossible to form a composite of a real and a complex matrix algebra that behaves correctly with regard to pure maps.

Remark 6.2. This theorem does not mention the faithfulness of this functor. If $\mathbb{E}$ satisfies local tomography, then in the same way as in Theorem 5.24 we see that it is faithful, but we expect that faithfulness already holds if $\mathbb{E}$ merely satisfies tomography. To determine this however we would need to show that the functor $F$ is strongly monoidal which does not seem trivial. We also expect that if $\mathbb{E}$ satisfies local tomography that the effect spaces of $\mathbb{E}$ must be isomorphic to those of complex instead of real $C^{*}$-algebras, but again, to prove this it seems like we need to know more about $\mathbb{E}$. We leave this as an open question for future work.

Remark 6.3. It should be noted that $\boldsymbol{v} \boldsymbol{N A}_{c p s u}^{\text {op }}$, the opposite category of von Neumann algebras with completely positive sub-unital maps, is also a monoidal PET [50, 51], but since it contains infinite-dimensional system it is not an operational PET. Finding suitable conditions under which we retrieve $\boldsymbol{v} \boldsymbol{N} \boldsymbol{A}_{c p s u}^{\text {op }}$ (or the bigger category of $J B W$-algebras and positive maps) is still an open question.

\section{Acknowledgments}

The author would like to thank Bas and Bram Westerbaan for all the useful and insightful conversations regarding effect algebras and order unit spaces and also for spotting some errors in the original proofs. Thanks also go out to Aleks Kissinger for helpful suggestions regarding the presentation of the work. Finally, the author thanks the anonymous reviewers for their helpful comments, in particular one reviewer who pointed out some flaws in the original use of (local) tomography. This work was supported by the ERC under the European Union's Seventh Framework Programme (FP7/2007-2013) / ERC grant n 320571 and by AFOSR grant FA2386-18-1-4028. 


\section{Appendix}

\section{A Operational Effect Theories}

It is a matter of routine to check that the opposite category $\mathbf{O U} \mathbf{S}^{\text {op }}$ forms an effect theory where the effects correspond to elements in $[0,1]_{V}$. Now since the states living in the continuous dual space $V^{*}$ order-separate $V$ [2, Corollary 1.27] we see that $\mathbf{O U S}{ }^{\text {op }}$ in fact forms an operational effect theory.

Proposition A.1. Let $\mathbf{E}$ be an effect theory with real scalars and where the unital states orderseparate the effects. Then there are no infinitesimal effects, i.e. for $p, q \in E f f(A)$, if $\frac{1}{2} p \leq \frac{1}{2}(q+1 / n)$ for all $n \in \mathbb{N}_{>0}$ then $p \leq q$.

Proof. Let $p$ be an effect such that $\frac{1}{2} p \leq \frac{1}{2}(q+1 / n)$ for all $n \in \mathbb{N}_{>0}$. Then for all unital states $\omega$ we will have $\left(\frac{1}{2} p\right) \circ \omega \leq \frac{1}{2}(q+1 / n) \circ \omega=\left(\frac{1}{2} q\right) \circ \omega+1 /(2 n)(1 \circ \omega)$. As this must hold for all $n$ we conclude that $\left(\frac{1}{2} p\right) \circ \omega \leq\left(\frac{1}{2} q\right) \circ \omega$. By assumption the unital states order-separate the effects so that we conclude that $\frac{1}{2} p \leq \frac{1}{2} q$ and hence $p \leq q$.

Definition A.2. We call an effect algebra $E$ an effect module (better known as a convex effect algebra) when there is an action of the real unit interval $[0,1]$ on $E$ such that $1 \cdot e=e, 0 \cdot e=0$, $(x+y) \cdot e=x \cdot e+y \cdot e$ and $(x y) \cdot e=x \cdot(y \cdot e)$. Such an action is necessarily unique [26]. We let Emod denote the category of effect modules with maps that preserve addition and scalar

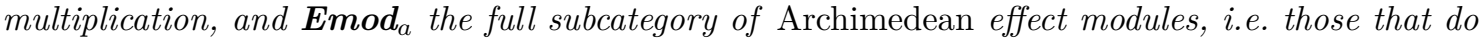
not have infinitesmal effects as defined in the previous proposition.

In an operational effect theory the sets of effects form effect modules, because when we have an effect $q: A \rightarrow I$ and a scalar $s: I \rightarrow I$, we get a new effect $s \circ q$ by composition. It can easily be checked that this action satisfies the properties necessary to be an effect module.

Proposition A.3. [26, 34] There is a one-to-one correspondence between Archimedean effect modules and order unit spaces: if $E$ is an Archimedean effect module, then we can find an order unit space $V$ such that $E \cong[0,1]_{V}$.

Proof of Theorem 2.11. There is the obvious effects functor for an effect theory Eff: $\mathbb{E} \rightarrow$ Emod $^{\text {op }}$ that maps each object to its set of effects and transformations $f: A \rightarrow B$ to $f^{*}: \operatorname{Eff}(B) \rightarrow$ $\operatorname{Eff}(A)$ given by $f^{*}(q)=q \circ f$. In an operational effect theory the sets of effects form Archimedean effect modules so that the image of this functor lands in $\mathbf{E m o d}_{a}$. We know by Ref. [34] that there is an equivalence of categories $F: \mathbf{E m o d}_{a} \rightarrow$ OUS, so that $F^{\text {op }} \circ$ Eff $: \mathbb{E} \rightarrow$ OUS ${ }^{\text {op }}$. It is then straightforward to see that the faithfulness of this functor exactly corresponds to local tomography of $\mathbb{E}$.

\section{B Proof of Theorem 5.24}

We will use a theorem by Alfsen and Shultz ([1, Theorem 9.33]) to prove Theorem 5.24. Their proof relies on a type of operators they also call compressions. Their compressions will turn out to be our assert maps. To distinguish our compressions from theirs, we will call their compressions AS-compressions.

Definition B.1. Let $P: A \rightarrow A$ be a map in an effect theory. We call it an $\boldsymbol{A} \boldsymbol{S}$-compression when $P$ is idempotent and it is bicomplemented: there exists an idempotent $Q: A \rightarrow A$ such that for all effects $q$ and states $\omega$ the following implications hold:

- $q \circ P=q \Longleftrightarrow q \circ Q=0$.

- $q \circ Q=q \Longleftrightarrow q \circ P=0$.

- $P \circ \omega=\omega \Longleftrightarrow Q \circ \omega=0$.

- $Q \circ \omega=\omega \Longleftrightarrow P \circ \omega=0$. 
Proposition B.2. In a PET where the effects separate the states (i.e. where $\omega=\omega^{\prime}$ when $p \circ$ $\omega=p \circ \omega^{\prime}$ for all effects $p$ ) the assert map asrt ${ }_{p}$ of a sharp effect $p$ is an AS-compression with bicomplement asrt $_{p^{\perp}}$.

Proof. In Proposition 4.11 it was already shown that assert maps of sharp effects are idempotent so it remains to show that they are bicomplemented. The bicomplement of $\operatorname{asrt}_{p}$ will turn out to be $\operatorname{asrt}_{p^{\perp}}$.

We have $q=q$ oasrt $_{p} \Longleftrightarrow q \leq p=\left(p^{\perp}\right)^{\perp} \Longleftrightarrow q \circ$ asrt $_{p^{\perp}}=0$ by an application of Propositions 4.11 and 4.13. Obviously the same holds with $p$ and $p^{\perp}$ interchanged.

Let $\omega$ be a state. Suppose $\operatorname{asrt}_{p} \circ \omega=\omega$ then $q \circ \operatorname{asrt}_{p^{\perp}} \circ \omega=q \circ \operatorname{asrt}_{p^{\perp}} \circ \operatorname{asrt}_{p} \circ \omega \leq p^{\perp} \circ \operatorname{asrt}_{p} \circ \omega=$ $0 \circ \omega=0$ for all effects $q$. Because the effects separate the states we can conclude that asrt $p_{p^{\perp}} \circ \omega=0$. Now for the other direction suppose $\operatorname{asrt}_{p^{\perp}} \circ \omega=0$, then $0=1 \circ \operatorname{asrt}_{p^{\perp}} \circ \omega=p^{\perp} \circ \omega$ so that $1 \circ \omega=\left(p+p^{\perp}\right) \circ \omega=p \circ \omega$. Then by definition of images: $\operatorname{im}(\omega) \leq p$ so that by Proposition $4.11 \operatorname{asrt}_{p} \circ \omega=\omega$. The other direction we get by interchanging $p$ and $p^{\perp}$. So asrt $p$ and asrt $p^{\perp}$ are indeed bicomplemented.

The results of Ref. [1] require a few concepts from convex geometry, in particular the notion of a face. Even though the definition we give below might seem foreign, it in fact corresponds with the intuitive idea of an extreme face of a convex set.

Definition B.3. Let $W$ be a real vector space and let $K \subseteq W$ be a convex subset. A face $F$ of $K$ is a convex subset such that whenever $\lambda x+(1-\lambda) y \in F$ with $0<\lambda<1$ then $x, y \in F$. Any extreme point of $K$ forms a face on its own. A face is called norm exposed when there exists a bounded affine positive functional $f: K \rightarrow \mathbb{R}^{+}$such that $f(a)=0 \Longleftrightarrow a \in F$. A face is called projective when there exists an AS-compression $P: W \rightarrow W$ such that for all $x \in K$ we have $P(x)=x \Longleftrightarrow x \in F$.

Lemma B.4. Let $A$ be a system in an operational PET. Any norm-exposed face of $S t(A)$ is projective.

Proof. Let $V$ be the vector space associated to $A$. Let $F \subseteq \operatorname{St}(A)$ be a norm-exposed face. That means that there is a positive affine functional $f: \operatorname{St}(A) \rightarrow \mathbb{R}_{\geq 0}$ with $f(\omega)=0 \Longleftrightarrow \omega \in F$. As $\operatorname{St}(A)$ is the state space of an order unit space, it forms a base of the base norm space $V^{*}[2$, Theorem 1.19]. As a result, its span is the entire positive cone of $V^{*}$ [2, Definition 1.10], and $f$ extends uniquely to a positive linear map $f: V^{*} \rightarrow \mathbb{R}[2$, Proposition 1.11]. In finite dimension we of course have $V \cong V^{*}$ so that there must be a $q \geq 0$ in $V$ such that $\forall \omega \in \operatorname{St}(A): f(\omega)=\omega(q)$. We can rescale $q$ without changing the zero set, so we can take $q$ to be an effect. By Proposition 4.6 $\omega(q)=0 \Longleftrightarrow \omega(\lceil q\rceil)=0$, so $q$ can be replaced by a sharp effect without changing the zero set. Now $\omega \in F \Longleftrightarrow \omega(q)=0 \Longleftrightarrow \operatorname{im}(\omega) \leq q^{\perp} \Longleftrightarrow \operatorname{asrt}_{q^{\perp}} \circ \omega=\omega$. Since assert maps of sharp effects are AS-compressions we see that $F$ is indeed projective.

Corollary B.5. Let $A$ be a system in an operational PET. Any AS-compression is an assert map.

Proof. If we have a AS-compression $P$ with complement $Q$ then we can construct $f: \operatorname{St}(A) \rightarrow \mathbb{R}^{+}$ by $f(\omega)=\|Q(\omega)\|$ which is affine (this is a standard result for the norm on base norm spaces in order separation with an order unit space [1]). Now obviously $f(v)=0 \Longleftrightarrow Q(v)=0 \Longleftrightarrow P(v)=v$, so we see that the projective face generated by $P$ is also norm exposed. But then by the previous proposition it is also the projective face of some assert map which necessarily must have the same projective unit. Because the projective unit determines the compression uniquely we see that $P$ must be equal to this assert map.

Lemma B.6. In an operational PET, the AS-compression of a convex extremal state is proportional to a convex extremal state.

Proof. By Corollary 5.23 convex extremal states are precisely the pure states. The only AScompressions are the assert maps of sharp effects, and assert maps are pure maps. By P2 the composition of pure maps is again pure, so that an AS-compression sends a convex extremal state to a pure state, which again by Corollary 5.23 must be convex extremal. 
Theorem 9.33 of [1] states that a state-space is isomorphic to that of a Jordan algebra when the following conditions are met:

- Every norm exposed face is projective (Lemma B.4).

- The convex extremal points span the space (Corollary 5.22).

- It satisfies symmetry of transition probabilities (Proposition 5.18).

- AS-compressions preserve convex extremal states (Lemma B.6).

Hence, we can indeed conclude that our systems are Euclidean Jordan algebras.

\section{Proof of Theorem 6.1}

As it has already been established that the effect spaces of systems in a operational PET correspond to Euclidean Jordan algebras, we will let $V$ and $W$ denote EJAs that should be understood to come from an operational PET. The monoidal structure of the PET obviously lifts to a bilinear map $V \times W \rightarrow V \otimes W$ where $V \otimes W$ is some other EJA corresponding to a system of the PET (not necessarily related to the regular vector space tensor product). In particular, if we have $v \geq 0$ in $V$ and $w \geq 0$ in $W$ then $v \otimes w \geq 0$ in $V \otimes W$.

Proposition C.1. In a monoidal operational PET the following are true.

1. A composite of pure maps is again pure.

2. A composite of normalized states is again a normalized state.

3. A composite of atomic effects is again an atomic effect.

4. For atomic effects $p$ and $q$ we have $\omega_{p} \otimes \omega_{q}=\omega_{p \otimes q}$.

5. If $q_{1} \perp p_{1}$ and $q_{2} \perp p_{2}$ are atomic orthogonal effects, then $q_{1} \otimes q_{2} \perp p_{1} \otimes p_{2}$.

Proof.

1. By definition of a monoidal PET.

2. Given two normalized states $\omega_{1}$ and $\omega_{2}$ we see that $1 \circ\left(\omega_{1} \otimes \omega_{2}\right)=(1 \otimes 1) \circ\left(\omega_{1} \otimes \omega_{2}\right)=$ $\left(1 \circ \omega_{1}\right) \otimes\left(1 \circ \omega_{2}\right)=1 \otimes 1=1$.

3. Let $p$ and $q$ be atomic effects. By Proposition 5.13 this is equivalent to them being sharp and pure. By point 1 we know that $p \otimes q$ is also pure and hence $p \otimes q$ must be proportional to an atom: $p \otimes q=\lambda r$. We calculate $1=(p \otimes q) \circ\left(\omega_{p} \otimes \omega_{q}\right)=(\lambda r) \circ\left(\omega_{p} \otimes \omega_{q}\right) \leq(\lambda 1) \circ\left(\omega_{p} \otimes \omega_{q}\right)=\lambda$, and hence $\lambda=1$ and we are done.

4. We know that $p \otimes q$ is atomic, and we know that $\omega_{p} \otimes \omega_{q}$ is a unital pure state (since it is a composite of pure unital states). We of course have $(p \otimes q) \circ\left(\omega_{p} \otimes \omega_{q}\right)=1$, but by Proposition 5.14 , the state $\omega_{p \otimes q}$ is the unique state with this property and hence $\omega_{p \otimes q}=\omega_{p} \otimes \omega_{q}$.

5. By Proposition 5.16, atomic effects $p$ and $q$ are orthogonal if and only if $q \circ \omega_{p}=0$. So supposing that $q_{1} \perp p_{1}$ and $q_{2} \perp p_{2}$ we calculate $\left(q_{1} \otimes q_{2}\right) \circ \omega_{p_{1} \otimes p_{2}}=\left(q_{1} \otimes q_{2}\right) \circ\left(\omega_{p_{1}} \otimes \omega_{p_{2}}\right)=$ $\left(q_{1} \circ \omega_{p_{1}}\right) \otimes\left(q_{2} \circ \omega_{p_{2}}\right)=0 \otimes 0=0$.

Definition C.2. The rank of an EJA $V$, denoted by rnk $V$, is equal to the maximal size of any set of orthogonal atomic effects in $V$.

The rank of the $n \times n$ matrix algebra $M_{n}^{s a}(F)$ is equal to $n$. The rank of a spin-factor is always equal to 2. Note that the size of a set of of orthogonal atomic effects $\left\{p_{i}\right\}$ is equal to the rank of the space if and only if $\sum_{i} p_{i}=1$.

Proposition C.3. Rank is preserved by compositing: rnk $V \otimes W=r n k V r n k W$. We also have $\operatorname{dim} V \otimes W \geq \operatorname{dim} V \operatorname{dim} W$. 
Proof. Let $\left\{p_{i}\right\}$ be a maximal set of orthogonal atomic effects in $V$, and let $\left\{q_{j}\right\}$ be a maximal set of orthogonal atomic effects in $W$. By maximality we must have $\sum_{i} p_{i}=1_{V}$ and $\sum_{j} q_{j}=1_{W}$. By Proposition C.1 the set $\left\{p_{i} \otimes q_{j}\right\}$ also consists of orthogonal atomic effects. Furthermore $\sum_{i, j} p_{i} \otimes q_{j}=\left(\sum_{i} p_{i}\right) \otimes\left(\sum_{j} q_{j}\right)=1_{V} \otimes 1_{W}=1_{V \otimes W}$ so that this set must also be maximal.

For the second part let $\left\{p_{i}\right\}$ be a basis of atomic effects of $V$ and similarly let $\left\{q_{j}\right\}$ be a basis of atomic effects in $W$. Suppose that $\operatorname{dim} V \otimes W<\operatorname{dim} V \operatorname{dim} W$, then $\left\{p_{i} \otimes q_{j}\right\}$ must be linearly dependent in $V \otimes W$. We will show that this leads to a contradiction. If this set is linear dependent, than without loss of generality we can write $p_{1} \otimes q_{1}=\sum_{i, j} \lambda_{i j} p_{i} \otimes q_{j}$, for some real numbers $\lambda_{i j}$ where the sum goes over all $i, j$ except $i=j=1$. Let $\omega$ be an arbitrary unital state on $W$ and apply the map id $\otimes \omega$ to both sides to get $p_{1} \otimes \omega\left(q_{1}\right)=\omega\left(q_{1}\right) p_{1}=$ $\sum_{i, j} \lambda_{i j} p_{i} \omega\left(q_{j}\right)=\sum_{i} p_{i}\left(\sum_{j} \lambda_{i j} \omega\left(q_{j}\right)\right)=p_{1}\left(\sum_{j} \lambda_{1 j} \omega\left(q_{j}\right)\right)+\sum_{i>1} p_{i}\left(\sum_{j} \lambda_{i j} \omega\left(q_{j}\right)\right.$. Rewrite this to $p_{1}\left(\omega\left(q_{1}\right)-\sum_{j} \lambda_{1 j} \omega\left(q_{j}\right)\right)=\sum_{i>1} p_{i} \sum_{j} \lambda_{i j} \omega\left(q_{j}\right)$. Since by assumption the $p_{i}$ are linearly independent this can only hold when $\sum_{j} \lambda_{i j} \omega\left(q_{j}\right)=\omega\left(\sum_{j} \lambda_{i j} q_{j}\right)=0$ for all states $\omega$ and $i>1$ and hence we must have $\sum_{j} \lambda_{i j} q_{j}=0$. Since the $q_{j}$ are also linearly independent this shows that $\lambda_{i j}=0$ when $i>1$. By interchanging the role of $p_{1}$ and $q_{1}$ we also get $\lambda_{i j}=0$ when $j>1$, so that the only nonzero value could be $\lambda_{11}$, which finishes the contradiction.

Proposition C.4. If $V$ and $W$ are simple than their composite $V \otimes W$ is also simple.

Proof. We know that the composite $V \otimes W$ has rank rnk $V \operatorname{rnk} W$, so if we can show that $V \otimes W$ must contain a simple factor of this rank than we are done.

Let $\left\{p_{i}\right\}$ be a maximal orthogonal set of atoms in $V$ and let $\left\{q_{j}\right\}$ be a maximal orthogonal set of atoms in $W$. Let $p$ be an atom in $V$ such that $\omega_{p}\left(p_{i}\right) \neq 0$ for all $i$, and similarly let $q$ be in $W$ such that $\omega_{w}\left(w_{j}\right) \neq 0$ (that such an atom can always be found can be straightforwardly verified by doing a case distinction on the possible simple factors). Then $\left(\omega_{p \otimes q}\right)\left(p_{i} \otimes q_{j}\right)=\omega_{p}\left(p_{i}\right) \omega_{q}\left(q_{j}\right) \neq 0$. As a result we conclude that $p \otimes q$ must belong to the same simple factor as all the $p_{i} \otimes q_{j}$, and hence this simple factor must have rank at least rnk $V$ rnk $W$.

Proposition C.5. Let $V$ be simple, then $V=M_{n}^{s a}(F)$ with $F=\mathbb{R}$ or $F=\mathbb{C}$.

Proof. This can be shown by a simple case distinction and dimension counting argument. We will work out one specific case, the other ones follow similarly. Suppose $V=M_{n}^{s a}(\mathbb{H})$. By Propositions C.3 and C.4 we then know that $V \otimes V$ must be a simple EJA with rank $n^{2}$ and $\operatorname{dim}(V \otimes V) \geq$ $\operatorname{dim}(V)^{2}$. The simple EJA of rank $n^{2}$ with the highest dimension is $M_{n^{2}}^{s a}(\mathbb{H})$. When $n>1$ however, the dimension of this space is still lower than $\operatorname{dim}\left(M_{n}^{s a}(\mathbb{H})\right)^{2}$, and hence such an EJA does not exist.

Note that the spin-factors $S_{2}$ and $S_{3}$ do allow the right sort of composites, but that these are in fact isomorphic to respectively real and complex matrix algebras.

Proposition C.6. Let $V$ and $W$ be simple EJAs, then both $V$ and $W$ are real matrix algebras, or both of them are complex matrix algebras.

Proof. By the previous proposition we know that they both must be real or complex, so that the only thing we need to show is that it cannot be that $V$ is complex while $W$ is real.

Let $V=M_{n}^{s a}(\mathbb{C})$ and $W=M_{m}^{s a}(\mathbb{R})$. By dimension counting and the previous proposition we know that $V \otimes W=M_{n m}^{s a}(\mathbb{C})$. Let $\omega$ be a pure state on $V$. The identity map on $W$ is of course pure, so that $\omega \otimes$ id $: M_{n m}^{s a}(\mathbb{C}) \rightarrow M_{m}^{s a}(\mathbb{R})$ is also a pure map. The study of pure maps on EJAs in [49] shows however that all quotient and comprehension spaces of EJAs are isomorphic to certain subalgebras of the space. In particular, if $f: M_{k}^{s a}(\mathbb{C}) \rightarrow W$ is a pure map, then $W \cong M_{l}^{s a}(\mathbb{C})$. The only time when $M_{m}^{s a}(\mathbb{R}) \cong M_{l}^{s a}(\mathbb{C})$ is when $m=l=1$, and hence the proposition is proved.

Finally, we can prove Theorem 6.1. Let $A$ be a system in a monoidal operational PET. By Theorem 5.24 we know that $\operatorname{Eff}(A)$ is isomorphic to the unit interval of a Euclidean Jordan algebra. Let $c$ be a minimal central element of this EJA, that hence corresponds to a simple factor of the algebra. The filter space associated to $c$ is then a simple algebra, and hence by Proposition C.5 it must be a real or complex matrix algebra. let $d$ be another minimal central element. Then its associated simple factor is also a real or complex matrix algebra and furthermore this algebra is associated to some system in the PET. By Proposition C.6 we can then conclude that either both the simple factors associated to $c$ and $d$ are real, or they are complex. Since this holds 
for all the simple factors we conclude that indeed $\operatorname{Eff}(A)$ is isomorphic to the unit interval of a real or complex $\mathrm{C}^{*}$-algebra. That all the systems in the OET must be all real or all complex follows similarly. Hence the functor of Theorem 5.24 restricts to the category of real or complex $\mathrm{C}^{*}$-algebras.

\section{References}

[1] Erik M Alfsen and Frederic W Shultz. Geometry of state spaces of operator algebras. Springer Science \& Business Media, 2012. DOI: https://doi.org/10.1007/978-1-4612-0019-2.

[2] Erik M Alfsen and Frederik W Shultz. State spaces of operator algebras: basic theory, orientations, and $C^{*}$-products. Springer Science \& Business Media, 2012. DOI: https://doi.org/10.1007/978-1-4612-0147-2.

[3] Howard Barnum, Jonathan Barrett, Matthew Leifer, and Alexander Wilce. Generalized no-broadcasting theorem. Physical Review Letters, 99(24):240501, 2007. DOI: https://doi.org/10.1103/PhysRevLett.99.240501.

[4] Howard Barnum, Markus P Müller, and Cozmin Ududec. Higher-order interference and singlesystem postulates characterizing quantum theory. New Journal of Physics, 16(12):123029, 2014. DOI: https://doi.org/10.1088/1367-2630/16/12/123029.

[5] Howard Barnum, Matthew Graydon, and Alexander Wilce. Composites and categories of Euclidean Jordan algebras. 2016. arxiv.org/1606.09331.

[6] Jonathan Barrett. Information processing in generalized probabilistic theories. Physical Review A, 75(3):032304, 2007. DOI: https://doi.org/10.1103/PhysRevA.75.032304.

[7] Jonathan Barrett, Niel de Beaudrap, Matty J Hoban, and Ciarán M Lee. The computational landscape of general physical theories. npj Quantum Information, 5(1):41, 2019.

[8] Mary K Bennett and David J Foulis. Interval and scale effect algebras. Advances in Applied Mathematics, 19(2):200-215, 1997. DOI: https://doi.org/10.1006/aama.1997.0535.

[9] Fernando GSL Brandao, Michał Horodecki, Jonathan Oppenheim, Joseph M Renes, and Robert W Spekkens. Resource theory of quantum states out of thermal equilibrium. Physical Review Letters, 111(25):250404, $2013 . \quad$ DOI: https://doi.org/10.1023/B:OPSY.0000047566.72717.71.

[10] Giulio Chiribella and Carlo Maria Scandolo. Operational axioms for diagonalizing states. In Chris Heunen, Peter Selinger, and Jamie Vicary, editors, Proceedings of the 12th International Workshop on Quantum Physics and Logic, Oxford, U.K., July 15-17, 2015, volume 195 of Electronic Proceedings in Theoretical Computer Science, pages 96-115. Open Publishing Association, 2015. DOI: https://doi.org/10.4204/EPTCS.195.8.

[11] Giulio Chiribella and Carlo Maria Scandolo. Purity in microcanonical thermodynamics: a tale of three resource theories. 2016. arxiv.org:1608.04460.

[12] Giulio Chiribella, Giacomo Mauro D'Ariano, and Paolo Perinotti. Probabilistic theories with purification. Physical Review A, 81(6):062348, 2010. DOI: https://doi.org/10.1103/PhysRevA.81.062348.

[13] Giulio Chiribella, Giacomo Mauro D'Ariano, and Paolo Perinotti. Informational derivation of quantum theory. Physical Review A, 84(1):012311, 2011. DOI: https://doi.org/10.1103/PhysRevA.84.012311.

[14] Kenta Cho, Bart Jacobs, Bas Westerbaan, and Abraham Westerbaan. An introduction to effectus theory. 2015. arxiv.org:1512.05813.

[15] Kenta Cho, Bart Jacobs, Bas Westerbaan, and Bram Westerbaan. Quotient-comprehension chains. In Chris Heunen, Peter Selinger, and Jamie Vicary, editors, Proceedings of the 12th International Workshop on Quantum Physics and Logic, Oxford, U.K., July 15-17, 2015, volume 195 of Electronic Proceedings in Theoretical Computer Science, pages 136-147. Open Publishing Association, 2015. DOI: https://doi.org/10.4204/EPTCS.195.10.

[16] Oscar Cunningham and Chris Heunen. Purity through factorisation. In Bob Coecke and Aleks Kissinger, editors, Proceedings 14th International Conference on Quantum Physics and Logic, Nijmegen, The Netherlands, 3-7 July 2017, volume 266 of Electronic Proceedings in Theoretical Computer Science, pages 315-328. Open Publishing Association, 2018. DOI: https://doi.org/10.4204/EPTCS.266.20. 
[17] Borivoje Dakic and Caslav Brukner. Quantum theory and beyond: is entanglement special? 2009. arxiv:0911.0695.

[18] Igor Devetak. Distillation of local purity from quantum states. Physical Review A, 71(6): 062303, 2005. DOI: https://doi.org/10.1007/BF01646490.

[19] Pau Enrique Moliner, Chris Heunen, and Sean Tull. Space in monoidal categories. In Bob Coecke and Aleks Kissinger, editors, Proceedings 14th International Conference on Quantum Physics and Logic, Nijmegen, The Netherlands, 3-7 July 2017, volume 266 of Electronic Proceedings in Theoretical Computer Science, pages 399-410. Open Publishing Association, 2018. DOI: https://doi.org/10.4204/EPTCS.266.25.

[20] Daniel I Fivel. Derivation of the rules of quantum mechanics from information-theoretic axioms. Foundations of Physics, 42(2):291-318, 2012. DOI: https://doi.org/10.1007/s10701011-9603-y.

[21] David J Foulis and Mary K Bennett. Effect algebras and unsharp quantum logics. Foundations of physics, 24(10):1331-1352, 1994. DOI: https://doi.org/10.1007/BF02283036.

[22] Christopher A Fuchs. Quantum mechanics as quantum information (and only a little more). 2002. arxiv:0205039.

[23] Stefano Gogioso. Fantastic quantum theories and where to find them. 2017.

[24] Stefano Gogioso and Fabrizio Genovese. Towards quantum field theory in categorical quantum mechanics. In Bob Coecke and Aleks Kissinger, editors, Proceedings 14th International Conference on Quantum Physics and Logic, Nijmegen, The Netherlands, 3-7 July 2017, volume 266 of Electronic Proceedings in Theoretical Computer Science, pages 349-366. Open Publishing Association, 2018. DOI: https://doi.org/10.4204/EPTCS.266.22.

[25] Philip Goyal. From information geometry to quantum theory. New Journal of Physics, 12(2): 023012, 2010. DOI: https://doi.org/10.1088/1367-2630/12/2/023012.

[26] Stanley Gudder. Convex structures and effect algebras. International Journal of Theoretical Physics, 38(12):3179-3187, 1999.

[27] Jack Gunson. On the algebraic structure of quantum mechanics. Communications in mathematical physics, 6(4):262-285, 1967. DOI: https://doi.org/10.1007/BF01646019.

[28] Harald Hanche-Olsen. JB-algebras with tensor products are $\mathrm{C}^{*}$-algebras. In Operator Algebras and their Connections with Topology and Ergodic Theory, pages 223-229. Springer, 1985.

[29] Lucien Hardy. Quantum theory from five reasonable axioms. 2001. arXiv:0101012.

[30] Lucien Hardy. Reconstructing quantum theory. In Quantum Theory: Informational Foundations and Foils, pages 223-248. Springer, 2016. DOI: https://doi.org/10.1007/978-94-0177303-4 7.

[31] Lucien Hardy and William K Wootters. Limited holism and real-vector-space quantum theory. Foundations of Physics, 42(3):454-473, 2012. DOI: https://doi.org/10.1007/s10701-011-96166.

[32] Chris Heunen and Bart Jacobs. Quantum logic in dagger kernel categories. Order, 27(2): 177-212, 2010. DOI: https://doi.org/10.1007/s11083-010-9145-5.

[33] Philipp Andres Höhn. Toolbox for reconstructing quantum theory from rules on information acquisition. Quantum, 1:38, 2017. DOI: https://doi.org/10.22331/q-2017-12-14-38.

[34] Bart Jacobs, Jorik Mandemaker, and Robert Furber. The expectation monad in quantum foundations. Information and Computation, 250:87-114, 2016. DOI: https://doi.org/10.1016/j.ic.2016.02.009.

[35] Pascual Jordan. Über Verallgemeinerungsmöglichkeiten des Formalismus der Quantenmechanik. Weidmann, 1933.

[36] Pascual Jordan, John von Neumann, and Eugene Wigner. On an algebraic generalization of the quantum mechanical formalism. Annals of Mathematics, 35(1):29-64, 1934. ISSN 0003486X. DOI: https://doi.org/10.2307/1968117.

[37] Marius Krumm, Howard Barnum, Jonathan Barrett, and Markus P Müller. Thermodynamics and the structure of quantum theory. New Journal of Physics, 19(4):043025, 2017. DOI: https://doi.org/10.1088/1367-2630/aa68ef.

[38] Lluis Masanes and Markus P Müller. A derivation of quantum theory from physical requirements. New Journal of Physics, 13(6):063001, 2011. DOI: https://doi.org/10.1088/13672630/13/6/063001. 
[39] Lluis Masanes, Markus P Müller, David Pérez-García, and Remigiusz Augusiak. Entanglement and the three-dimensionality of the Bloch ball. Journal of Mathematical Physics, 55(12): 122203, 2014. DOI: https://doi.org/10.1063/1.4903510.

[40] Sandu Popescu. Nonlocality beyond quantum mechanics. Nature Physics, 10(4):264, 2014. DOI: https://doi.org/10.1038/nphys2916.

[41] John H Selby, Carlo Maria Scandolo, and Bob Coecke. Reconstructing quantum theory from diagrammatic postulates. 2018. arxiv:1802.00367.

[42] Anthony J Short and Stephanie Wehner. Entropy in general physical theories. New Journal of Physics, 12(3):033023, 2010. DOI: https://doi.org/10.1088/1367-2630/12/3/033023.

[43] Robert W Spekkens. Evidence for the epistemic view of quantum states: A toy theory. Physical Review A, 75(3):032110, 2007. DOI: https://doi.org/10.1103/PhysRevA.75.032110.

[44] Sean Tull. A categorical reconstruction of quantum theory. 2016. arxiv:1804.02265.

[45] Sean Tull. Categorical operational physics. 2019. arxiv:1902.00343.

[46] John van de Wetering. Sequential product spaces are Jordan algebras. Journal of Mathematical Physics, 60(6):062201, 2019. DOI: https://doi.org/10.1063/1.5093504.

[47] Abraham Westerbaan and Bas Westerbaan. A universal property for sequential measurement. Journal of Mathematical Physics, 57(9):092203, 2016. DOI: https://doi.org/10.1063/1.4961526.

[48] Abraham Westerbaan and Bas Westerbaan. Paschke dilations. In Ross Duncan and Chris Heunen, editors, Proceedings 13th International Conference on Quantum Physics and Logic, Glasgow, Scotland, 6-10 June 2016, volume 236 of Electronic Proceedings in Theoretical Computer Science, pages 229-244. Open Publishing Association, 2017. DOI: https://doi.org/10.4204/EPTCS.236.15.

[49] Abraham Westerbaan, Bas Westerbaan, and John van de Wetering. Pure Maps between Euclidean Jordan Algebras. In Peter Selinger and Giulio Chiribella, editors, Proceedings of the 15th International Conference on Quantum Physics and Logic, Halifax, Canada, 3-7th June 2018, volume 287 of Electronic Proceedings in Theoretical Computer Science, pages 345364. Open Publishing Association, 2019. DOI: https://doi.org/10.4204/EPTCS.287.19.

[50] Abraham A. Westerbaan. The Category of Von Neumann Algebras. PhD thesis, Radboud Universiteit Nijmegen, 2019. URL https://bram. westerbaan. name/thesis.pdf.

[51] Bas Westerbaan. Dagger and dilations in the category of von Neumann algebras. PhD thesis, Radboud Universiteit Nijmegen, 2019. URL https://westerbaan.name/ bas/thesis.pdf.

[52] Alexander Wilce. A royal road to quantum theory (or thereabouts). Entropy, 20(4):227, 2018. DOI: https://doi.org/10.3390/e20040227. 